\title{
INDUCTIVE CONSTRUCTION OF HOMOGENEOUS CONES
}

\author{
BY
}

JOSEF DORFMEISTER

\begin{abstract}
A method is explained how to construct all homogeneous cones in a unique way out of lower dimensional ones. The infinitesimal automorphisms of such a cone and its associated left-symmetric algebras are described in terms of the lower dimensional constituents of the cone. It is characterized when a homogeneous cone is self-dual or a sum of homogeneous cones.
\end{abstract}

In his paper, The construction of homogeneous convex cones [9] O. Rothaus used È. Vinberg's results on left-symmetric algebras [13] to show that every homogeneous regular cone can be constructed from a lower dimensional one. But there is no description of the infinitesimal automorphisms of the cone with respect to its low dimensional constituents and, as noted in [9], this construction is not unique.

Starting from [10] and [8] and using [4], [5] and [5a] this paper shows how to build up a homogeneous regular cone from lower dimensional ones in a unique way. Further there is a description of the Lie-algebra of infinitesimal automorphisms of a cone in terms of its building blocks. As a by-product we obtain a splitting theorem of left-symmetric algebras with respect to the lower dimensional constituents of the cone and several equivalent conditions for a cone to be self-dual. Finally, there is a canonical mapping shown to be injective, a result that will be helpful in investigations on homogeneous Siegel domains.

In detail, the organization of this paper is as follows. In $\$ 1$ we assemble most of those definitions and results of the literature that are basic for this paper. We also unify the notation used. Especially, we define triples $F=$ $\langle K, \eta, e\rangle$, where $K$ is a homogeneous regular cone, $\eta$ a function on $K$ and $e$ a point of $K$. For $F$ and a properly defined Lie-algebra we derive splitting theorems with respect to the lower dimensional constituents of $K$ in $\S \S 2,3$. Results on sums of cones and the characterization of the self-dual case are established in $\S 4$. The connections to È. Vinberg's left-symmetric algebras are described in $\$ \S 5,6$. In $\$ 7$ we are mainly interested in the question: When is $A_{1 / 2}$ injective? Before we arrive at the main result we present some lemmata

Received by the editors April 10, 1977 and, in revised form, February 15, 1978.

AMS (MOS) subject classifications (1970). Primary 22E60, 17C35.

Key words and phrases. Homogeneous cone, infinitesimal automorphism, Jordan algebra. 
that may be useful in later work on homogeneous Siegel domains. Finally, in $\S 8$, we show how to construct every homogeneous regular cone in a unique way out of lower dimensional ones. Here the "Construction Theorem" uses nearly all that has been deduced before.

This paper's contents are part of the author's thesis [3]. We mention that in [3] it was not generally assumed that all cones which appear are homogeneous. In this paper, however, for the sake of simplicity of notation and shortness of statements we concentrate on the investigation of homogeneous cones.

\section{Definitions and notations.}

1. In what follows $\mathscr{F}$ stands for the class of triples $\langle K, \eta, e\rangle$, where $K$ is a regular cone (see [5a]) in a finite-dimensional $\mathbf{R}$-vector space $V, e$ is a point of $K$ and $\eta$ is a positive real mapping $\eta: K \rightarrow \mathbf{R}^{+}$such that

(1.1) $\eta$ is infinitely differentiable,

(1.2) $\eta$ is homogeneous (there exists $k \in \mathbf{R}$ such that $\eta(\tau x)=\tau^{k} \eta(x)$ for all $\tau>0, x \in K)$,

(1.3) the bilinear mapping $(u, v) \mapsto \Delta_{x}^{u} \Delta_{x}^{v} \log \eta(x)$ is positive-definite, for all $x \in K$ (here $\Delta_{x}^{u}$ means differentiation at $x$ in direction $u$ ),

(1.4) for every sequence $x_{n}$ of $K$ that converges to a boundary point of $K$, the sequence $\eta\left(x_{n}\right)$ converges to $+\infty$,

(1.5) the group $\operatorname{Aut}(K, \eta):=\{W \in \mathrm{GL} V ; W K=K$, there exists an $\alpha(W)$ $>0$ such that $\eta(W x)=\alpha(W) \eta(x)$ for all $x \in K$ \} operates transitively on $K$.

For every triple $F=\left\langle K_{F}, \eta_{F}, e_{F}\right\rangle$ of $\mathscr{F}$ one puts $V=V_{F}$ and defines as in [4], [5a],

$(1.6) \sigma_{F}(u, v):=\left.\Delta_{x}^{u} \Delta_{x}^{v} \log \eta_{F}(x)\right|_{x=e_{F}}, u, v \in V_{F}$.

(By (1.3) the symmetric bilinearform $\sigma_{F}$ is positive-definite.)

(1.7) $h_{F}: K \rightarrow K^{\sigma_{F}}, \sigma_{F}\left(h_{F}(x), u\right):=-\Delta_{x}^{u} \log \eta_{F}(x)$.

(As usual, for a regular cone $K$ in $V$ and a nondegenerate bilinearform $\sigma$ on $V$, we put $K^{\sigma}:=\{x \in V ; \sigma(x, y)>0$ for all $0 \neq y \in \bar{K}$ (by $\bar{K}$ we denote the closure of $K$ in $V$ ) $\}$. The regular cone $K^{\sigma}$ in $V$ is called the $\sigma$-dual cone for $K$ (with respect to $\sigma$ ).)

(1.8) $H_{F}: K \rightarrow$ End $V_{F}, \sigma_{F}\left(H_{F}(x) u, v\right):=\Delta_{x}^{u} \Delta_{x}^{v} \log \eta_{F}(x)$,

(1.9) $A_{F}(u):=-\left.\frac{1}{2} \Delta_{x}^{u} H_{F}(x)\right|_{x=e_{F}}, u \in V_{F}$.

It is well known from [4] that $(u, v) \mapsto u v:=A_{F}(u) v$ defines a commutative algebra $\mathfrak{A}_{F}$ on $V_{F}$ with unit $e_{F}$ and that $h_{F}$ and $H_{F}$ are rational. We will of ten abbreviate $x \cdot y z:=x(y z)$ for the product of three elements in $\mathfrak{A}_{F}$.

In what follows the index $F$ is dropped when there is no possibility of confusion (this also applies to (1.14)). In $\S 1$, e.g., there is used only one triple $F$, therefore-except in definitions-we omit the index $F$. But at other places it is inevitable to index (see Lemma 2.2., Corollary 4.4, . . ).

Furthermore, one easily derives as in [4], [5a] 
(1.10) $H(x)=H(x)^{\sigma}$ is positive-definite for all $x \in K$,

(1.11) $A(u)=A(u)^{\sigma}, \sigma(u v, w)=\sigma(u, v w)$ for all $u, v, w \in V$,

(1.12) $h(W x)=\left[W^{\sigma}\right]^{-1} h(x)$ for all $x \in K, W \in \operatorname{Aut}(K, \eta)$,

(1.13) $H(W x)=\left[W^{\sigma}\right]^{-1} H(x) W^{-1}$ for all $x \in K, W \in \operatorname{Aut}(K, \eta)$.

Note that for (1.6)-(1.13) the property (1.5) of $\mathscr{F}$ is not used. We will take advantage of this fact in $\$ 2$.

2. Let $\mathfrak{B}$ be a commutative algebra with product $(u, v) \mapsto u v$ and left-multiplications $B(u)$. For $x \in \mathfrak{B}$ we define a new algebra $\mathfrak{B}_{x}$ on the underlying vectorspace of $\mathfrak{B}$ by the multiplication law $(u, v) \mapsto(u x) v+u(x v)-x(u v)$. The algebra $\mathfrak{B}_{x}$ is called the mutation of $\mathfrak{B}$ with respect to $x$; left-multiplication in $\mathfrak{B}_{x}$ is denoted by $B_{x}(u)$. We obviously have $B_{x}(u)=B(B(x) u)+$ $[B(u), B(x)]$ for all $x, u \in \mathfrak{B}$. In this paper we are mainly concerned with mutations of $\mathfrak{A}=\mathfrak{U}_{F}$.

Using the definitions above, and the notation Lie $\Gamma$ for the Lie-algebra of a closed subgroup $\Gamma$ of GL $V$, we put

$(1.14) \mathfrak{S}_{F}:=\left\{x \in V_{F} ; A_{x}(u) \in \operatorname{Lie} \operatorname{Aut}\left(K_{F}, \eta_{F}\right)\right.$ for all $\left.u \in V_{F}\right\}$.

We have the following important

THEOREM 1.1 [5a]. $\subseteq \neq 0$.

This means that to all algebras $\mathfrak{A}_{x}, x \in \mathcal{S}, x \neq 0$, we may apply the results of O. Rothaus [10]. Furthermore, we can use the theory of M. Koecher [8] in a nontrivial manner.

To unify notation we restate some theorems of [8] and [10].

THEOREM 1.2 [8]. (a) $A_{T^{o} b}(v)=A_{b}(T v)+\left[A_{b}(v), T\right]$ for all $T \in$ Lie $\operatorname{Aut}(K, \eta), v \in V, b \in \mathfrak{S}$.

(b) $\mathfrak{A}_{b}, b \in \mathfrak{S}$, is a Jordan-algebra.

(c) $T^{\sigma} \subseteq \subset \subseteq$ for all $T \in$ Lie $\operatorname{Aut}(K, \eta)$.

(d) $\subseteq$ is a formally-real Jordan-subalgebra of $\mathfrak{A}$.

From part (d) of the above theorem, one deduces that $\subseteq$ has a unit (see [1]) which we call $c$; for simplicity of notation we also use $c_{1}:=c, c_{0}:=e-c$.

Henceforth we reserve the letter $c$ for the unit of $\mathfrak{S}$.

We recall from [5a] that for a triple $F=\langle K, \eta, e\rangle$ one puts $\mathfrak{X}_{F}:=\{x \in$ $\left.V_{F} ; A_{F}(x) \in \operatorname{Lie} \operatorname{Aut}(K, \eta)\right\}$. As usual, we drop the index $F$ when there is no possibility of confusion.

REMARK 1.3. (1) From (1.14) one easily concludes $\subseteq \subset \mathfrak{X}$ (see [5a]). Therefore, for any complete system of idempotents (CSI) $d_{1}, \ldots, d_{r-1}$ of $\mathfrak{S}$ one gets a CSI $d_{1}, \ldots, d_{r}, d_{r}:=e-c$, of $\mathfrak{U}$ that is contained in $\mathfrak{X}$. Hence by [5a] one has a Peirce-decomposition of $\mathfrak{A}$ with respect to $d_{1}, \ldots, d_{r}$ the Peirce-spaces of which are pairwise orthogonal with respect to $\sigma$.

(For the definition of a CSI and a Peirce-decomposition see [5a, §3].) 
(2) Because of part (b) of Theorem 1.2, one may apply the results of [2, §2] to $\mathfrak{A}_{b}$ for $b \in \mathfrak{S}$ with $b^{2}=b$.

(3) In Theorem 1.2. we put $b=c, v=c$ and $T=A(x), x \in \mathfrak{S}$, and deduce $A_{x}(c)=A_{c}(x)+\left[A_{c}(c), A(x)\right]$. We use the definition of $\mathfrak{A}_{b}$ and get $A_{x}(c)=$ $A(x)+[A(c), A(x)], A_{c}(x)=A(x)+[A(x), A(c)]$ and $A_{c}(c)=A(c)$. Putting together these four expressions we derive $[A(x), A(c)]=0$. Furthermore, this implies $A_{c}(x)=A(x)$ for $x \in \widetilde{S}$.

Let $\mathfrak{B}$ be a commutative algebra. Then by Der $\mathfrak{B}$ we denote the set of derivations of $\mathfrak{B}$.

Lemma 1.4. (a) Lie $\operatorname{Aut}(K, \eta) \subset A(\Im)+\operatorname{Der} \mathfrak{A}_{c}$,

(b) Let $T \in \operatorname{Lie} \operatorname{Aut}(K, \eta)$ and $b \in \mathfrak{S}$. Then $T \in \operatorname{Der} \mathfrak{U}_{b} \Leftrightarrow T^{\sigma} b=0$.

Proof. Part (b) is immediately clear from Theorem 1.2. To prove (a) it therefore suffices to derive $\left[T-A_{c}\left(T^{\sigma} c\right)\right]^{\sigma} c=0$ for all $T \in \operatorname{Lie} \operatorname{Aut}(K, \eta)$. But by [2, Folgerung 1.2.c] we have $y:=\left[A_{c}\left(T^{\sigma} c\right)\right]^{\sigma} c=A_{r}(c) c, r:=T^{\sigma} c$, and from Theorem 1.2, we deduce $T^{\sigma} c \in \Im$ S. Hence $y=T^{\circ} c$ and the lemma is proved.

3. Now, let $p \in \Im, p^{2}=p$. By Remark 1.3, we form the Peirce-decomposition $\mathfrak{A}=\mathfrak{A}_{1}(p)+\mathfrak{A}_{1 / 2}(p)+\mathfrak{A}_{0}(p)$ of $\mathfrak{A}, \mathfrak{A}_{i}(p)=\{x \in \mathfrak{A} ; p x=i x\}$. We reduce $\mathfrak{A}_{i}(p)$ to $\mathfrak{A}_{i}$ when no confusion can arise. Further, without mentioning, for $x \in V$ we always use the expansion $x=x_{1}+x_{1 / 2}+x_{0}$ with $x_{i} \in \mathfrak{A}_{i}$. We systematically use the indices $0, \frac{1}{2}, 1$ to point out to which $\mathfrak{A}_{i}$ an element of $V$ belongs. We define

$$
A_{j}\left(x_{i}\right):=\left.A\left(x_{i}\right)\right|_{\mathfrak{A}_{j}}, \quad j=0, \frac{1}{2}, 1, i=1,0, x_{i} \in \mathfrak{U}_{i} .
$$

We note that $A_{j}\left(x_{i}\right)$ is an endomorphism of $\mathfrak{A}_{j}$ because of the composition rules of a Peirce-decomposition [5a].

Let $\pi_{i}, i=0, \frac{1}{2}, 1$, denote the projection of $\mathfrak{A}=\mathfrak{A}_{1}+\mathfrak{A}_{1 / 2}+\mathfrak{U}_{0}$ onto $\mathfrak{A}_{i}$. We put $K_{i}=K_{i}^{p}:=\pi_{i}(K), i=0,1$, and get from [9, IV, Theorem 17].

THEOREM 1.5 [10]. The cones $K_{1}$ and $K_{0}$ are regular cones lying in the closure $\bar{K}$ of $K$. Moreover, $A_{1}\left(x_{1}\right) \in$ Lie Aut $K_{1}$ for all $x_{1} \in \mathfrak{A}_{1}$.

In [10] there was also stated that $K_{1}$ is a homogeneous self-dual cone. We are going to sharpen this result.

For a formally-real Jordan-algebra $\mathfrak{B}$ we call the connected component of the set of invertible elements of $\mathscr{B}$ that contains the unit of $\mathscr{B}$ the positive cone of $\mathfrak{B}$. In $[1, \mathrm{XI}]$ this set has been denoted by $Y_{\mathfrak{B}}$. For detailed results for positive cones of formally-real Jordan-algebras we refer to [1] or [6].

THEOREM 1.6. $K_{1}$ is the positive cone of the formally-real Jordan-subalgebra $\mathfrak{U}_{1}$ of $\mathfrak{U}$. 
Proof. Put $\mathfrak{A}_{i}:=\mathfrak{A}_{i}(c)$. Then note that $D c \in \mathfrak{A}_{1 / 2}$ for all $D \in \operatorname{Der} \mathfrak{A}_{c}$ because of $D c=D\left(c^{2}\right)=2 c \cdot D c$. Further, $A_{c}\left(x_{1 / 2}\right) \in$ Der $\mathfrak{A}_{c}$ for all $x_{1 / 2} \in$ $\mathfrak{A}_{1 / 2}\left(\right.$ from Lemma 1.4(b)). Hence $\hat{D}:=D-2 A_{c}(D c) \in \operatorname{Der} \mathfrak{A}_{c} \cap \operatorname{Aut}(K, \eta)$ and $\hat{D} c=0$ for all $D \in \operatorname{Der} \mathfrak{A}_{c} \cap \operatorname{Aut}(K, \eta)$. We consider the set $\delta:=\{D \in$ $\left.\operatorname{Der} \mathfrak{A}_{c} \cap \operatorname{Aut}(K, \eta) ; D c=0\right\}$. It is easy to verify $D \mathfrak{A}_{i} \subset \mathfrak{A}_{i}, i=0, \frac{1}{2}, 1$, for all $D \in \delta$. Further, from Lemma $1.4(\mathrm{~b})$ we deduce $\delta \subset$ Der $\mathfrak{A}_{c}$. Hence $\mathfrak{a}:=\left\{A_{c}\left(x_{1}\right) ; x_{1} \in \mathfrak{A}_{1}\right\}+\mathfrak{d}$ is a Lie-subalgebra of Lie $\operatorname{Aut}(K, \eta)$ and from Lemma 1.4(a) we conclude Lie $\operatorname{Aut}(K, \eta)=a+\left\{A_{c}\left(x_{1 / 2}\right) ; x_{1 / 2} \in \mathfrak{A}_{1 / 2}\right\}$. It is clear that the elements of a leave $\mathfrak{A}_{1}$ invariant. Therefore $a_{1}:=\left\{A_{1}\left(x_{1}\right)\right.$; $\left.x_{1} \in \mathfrak{U}_{1}\right\}+\left\{D \mid \mathfrak{A}_{1} ; D \in \mathfrak{d}\right\}$ is a Lie-subalgebra of Lie Aut $K_{1}$. Furthermore, by (1.5), we know that the set $\left\{T\left(x_{1}+e-c\right) ; T \in \operatorname{Lie} \operatorname{Aut}(K, \eta)\right\}$ equals $V$ for all $x_{1} \in K_{1}$. Hence $\left\{T x_{1} ; T \in a_{1}\right\}$ equals $\mathfrak{U}_{1}$ for all $x_{1} \in K_{1}$. Therefore, the Lie-subgroup $\Omega_{1}$ of Aut $K_{1}$ which is generated by $a_{1}$ acts transitively on $K_{1}$. Now, it obviously suffices to prove that $\Omega_{1} c$ is the positive cone of the formally-real Jordan-algebra $\mathfrak{A}_{1}$. But the second summand of $a_{1}$ is contained in the isotropy algebra for $c \in K_{1}$ and consists of derivations of $\mathfrak{U}_{1}$. Hence $K_{1}$ equals the set of $W c$ where $W$ is a product of elements of the form $\exp A_{1}\left(x_{1}\right)$, $x_{1} \in \mathfrak{A}_{1}$. From [1, XI, Satz 2.4], we conclude that $K_{1}$ is the positive cone of $\mathfrak{U}_{1}$.

From the last theorem it follows that all $x_{1} \in K_{1}$ are invertible in $\mathfrak{A}_{1}$. Hence, see e.g. [2, §2], the endomorphism $A_{1 / 2}\left(x_{1}\right)$ of $\mathfrak{A}_{1 / 2}$ is invertible for all $x_{1} \in K_{1}$. This enables us to define

$$
\begin{aligned}
k_{1 / 2}^{p}: K \rightarrow \mathfrak{A}_{1 / 2}, \quad x \mapsto\left[A_{1 / 2}\left(x_{1}\right)\right]^{-1} x_{1 / 2}, \\
k_{0}^{p}: K \rightarrow \mathfrak{A}_{0}, \quad x \mapsto x_{0}-\frac{1}{2}(e-p)\left(x_{1 / 2} k_{1 / 2}^{p}(x)\right) .
\end{aligned}
$$

As usual we omit " $p$ " when no confusion can arise. Using these definitions and (1.14) we easily get

LEMMA 1.7. (a) Every $x \in K$ can be written in the form

$$
x=\exp A_{p}\left(k_{1 / 2}(x)\right)\left(x_{1}+k_{0}(x)\right), \quad x_{1} \in K_{1} .
$$

(b) $k_{0}(K) \subset K_{0}$.

Proof. For $x=x_{1}+x_{1 / 2}+x_{0} \in K$ we can form $k_{1 / 2}(x)$ and $k_{0}(x)$ as remarked above. We expand the right-hand side and get

$$
\begin{aligned}
\exp A_{p}\left(k_{1 / 2}(x)\right. & \left(x_{1}+k_{0}(x)\right) \\
= & x_{1}+k_{0}(x)+x_{1} k_{1 / 2}(x)+(e-p)\left(k_{1 / 2}(x) \cdot x_{1} k_{1 / 2}(x)\right) \\
= & x_{1}+x_{0}-(e-p)\left(x_{1 / 2} \cdot k_{1 / 2}(x)\right) \\
& +x_{1 / 2}+(e-p)\left(k_{1 / 2}(x) \cdot x_{1 / 2}\right)=x .
\end{aligned}
$$

Here we have used $A_{p}\left(x_{1 / 2}\right) y_{1 / 2}=(e-p)\left(x_{1 / 2} y_{1 / 2}\right)$, an identity which 
follows immediately from the definitions of $\mathfrak{A}_{p}$ and $\mathfrak{A}_{1 / 2}$. It remains to show $x_{1} \in K_{1}$ and $k_{0}(x) \in K_{0}$. But by the definition of $\subseteq$ we have for $p \in \subseteq$ especially $\exp A_{p}\left(k_{1 / 2}(x)\right) \in \operatorname{Aut}(K, \eta)$. Hence $x_{1}+k_{0}(x) \in K$. This, clearly, implies the assertion.

The next result gives a very useful description of $K$.

THEOREM 1.8. The mapping $K_{1} \times \mathfrak{A}_{1 / 2} \times K_{0} \rightarrow K, \quad\left(x_{1}, x_{1 / 2}, x_{0}\right) \mapsto$ $\exp A_{p}\left(x_{1 / 2}\right)\left(x_{1}+x_{0}\right)$ is a real-analytic diffeomorphism with functional determinant $\operatorname{det} A_{1 / 2}\left(x_{1}\right)$. The inverse mapping is given by $x \mapsto\left(x_{1}, k_{1 / 2}(x)\right.$, $\left.k_{0}(x)\right)$.

Proof. Put $\varphi\left(x_{1}, x_{1 / 2}, x_{0}\right):=\exp A_{p}\left(x_{1 / 2}\right)\left(x_{1}+x_{0}\right)$ and denote by $Y$ the range of $\varphi$. Obviously, $\varphi$ is real analytic. We have $K \subset Y$ by Lemma 1.7 and $Y \subset \bar{K}$, the closure of $K$ in $V$, because of $K_{1}+K_{0} \subset \bar{K}$ and $\exp A_{p}\left(x_{1 / 2}\right) \in$ $\operatorname{Aut}(K, \eta)$. Now, a simple computation shows that the functional determinant of $\varphi$ equals $\operatorname{det} A_{1 / 2}\left(x_{1}\right)$. From the remarks preceding (1.16) we know that $A_{1 / 2}\left(x_{1}\right)$ is invertible for $x_{1} \in K_{1}$. Hence $\varphi$ is an open map. But the open kernel of $\bar{K}$ equals $K$. Therefore $K=Y$ and $\varphi$ is surjective. Assume $\varphi(x)=$ $\varphi(y)$. Then we expand and get $x_{1}=y_{1}, x_{1} x_{1 / 2}=y_{1} y_{1 / 2}$ and $x_{0}+(e-$ $p)\left(x_{1 / 2} \cdot x_{1} x_{1 / 2}\right)=y_{0}+(e-p)\left(y_{1 / 2} \cdot y_{1} y_{1 / 2}\right)$. As remarked above, for $x_{1} \in$ $K_{1}$, the endomorphism $A_{1 / 2}\left(x_{1}\right)$ of $\mathfrak{A}_{1 / 2}$ is invertible. From this we easily derive $x=y$, i.e. $\varphi$ is injective. Finally, by Lemma 1.7(a), we conclude that $x \mapsto\left(x_{1}, k_{1 / 2}(x), k_{0}(x)\right)$ equals $\varphi^{-1}$.

From this result we derive two corollaries.

Corollary $1.9[10]$.

$$
\begin{aligned}
K & =\bigcup_{x_{1 / 2} \in \mathfrak{A}_{1 / 2}} \exp A_{p}\left(x_{1 / 2}\right)\left(K_{1}+K_{0}\right) \\
& =\left\{\exp A_{p}\left(x_{1 / 2}\right)\left(x_{1}+x_{0}\right) ; x_{1} \in K_{1}, x_{0} \in K_{0}, x_{1 / 2} \in \mathfrak{A}_{1 / 2}\right\} \\
& =\left\{x \in V ; x_{1} \in K_{1}, k_{0}(x) \in K_{0}\right\} .
\end{aligned}
$$

Proof. By Theorem 1.8 we, obviously, have only to prove the last equality. Let $x \in K$; then $x_{1} \in K_{1}$ and $k_{0}(x) \in K_{0}$ by Lemma 1.7. Assume, on the other hand, $x \in V$ and $x_{1} \in K_{1}$. As mentioned above, by this condition we may form $k_{1 / 2}(x)$ and $k_{0}(x)$. We use this and get $x=\exp A_{p}\left(k_{1 / 2}(x)\right) \cdot\left(x_{1}+\right.$ $\left.k_{0}(x)\right)$ as in the proof of Lemma 1.7. The assumption $k_{0}(x) \in K_{0}, x_{1} \in K_{1}$, implies $x \in K$ by Theorem 1.8 and the corollary is proved.

Corollary 1.10. $K_{1}+K_{0}=\left\{x \in K ; x_{1 / 2}=0\right\}$.

Proof. By Theorem 1.8. we know $K_{1}+K_{0} \subset K$. The corollary is obvious now. 
2. Splitting of $F$. In $\S 1$ we saw by Theorem 1.8 that $K$ "splits up" into cones $K_{1}$ and $K_{0}$. There we have $\mathfrak{A}_{1} \neq 0$ for $p=c$ because of Theorem 1.1, and $\mathfrak{A}_{0}=0$ if and only if $K_{1}=K$ is the positive cone of the formally-real Jordan-algebra $\mathfrak{A}_{1}=\mathfrak{A}$. So in general this splitting is nontrivial.

As in $\S 1$ let $F=\left\langle K_{F}, \eta_{F}, e_{F}\right\rangle$ be a triple of $\mathscr{F}$ and $p \in \mathfrak{S}_{F}, p^{2}=p$. We form again the Peirce-decomposition $\mathfrak{U}=\mathfrak{A}_{1}+\mathfrak{U}_{1 / 2}+\mathfrak{U}_{0}$ of $\mathfrak{A}=\mathfrak{A}_{F}$ with respect to $p$ and put $p_{1}:=p, p_{0}:=e-p$. We form the cones $K_{1}$ and $K_{0}$ with respect to $p$ and set

(2.1) $\eta_{1}\left(x_{1}\right):=\eta\left(x_{1}+p_{0}\right), x_{1} \in K_{1}$,

(2.2) $\eta_{0}\left(x_{0}\right):=\eta\left(p_{1}+x_{0}\right), x_{0} \in K_{0}$,

(2.3) $F_{\mathrm{i}}:=\left\langle K_{i}, \eta_{i}, p_{i}\right\rangle, i=0,1$.

To make clear with respect to which $p \in \mathbb{S}$ the Peirce-decomposition $\mathfrak{U}=\mathfrak{A}_{1}+\mathfrak{U}_{1 / 2}+\mathfrak{A}_{0}$ is formed one could put a superscript at $\eta_{i}$ and $F_{i}$. To avoid notational complications as far as possible we disregard this superscript. We hope that this will cause no confusion.

The situation is different for the subscript. Here we feel that indexing suggestively clarifies in which Peirce-space (with respect to $p$ ) we are working and easily helps to keep track of the two triples occurring.

The purpose of this paragraph is to point out the connections between $F$ and $F_{i}$. We start with an investigation of $\eta$.

LEMMA 2.1. (a) $\eta\left(x_{1}+x_{0}\right)=\alpha \eta_{1}\left(x_{1}\right) \eta_{0}\left(x_{0}\right), \alpha=[\eta(e)]^{-1}$, for all $x_{1} \in K_{1}$, $x_{0} \in K_{0}$.

(b) $\eta(x)=\eta\left(x_{1}+k_{0}(x)\right)$ for all $x \in K$.

(c) The functions $\eta_{i}: K_{i} \rightarrow \mathbf{R}^{+}$satisfy (1.1) to (1.4).

Proof. (a) For $x_{1} \in K_{1}$ we use Theorem 1.6. and find $y_{1} \in \mathfrak{U}_{1}$ such that $x_{1}=\exp A\left(y_{1}\right) p$. We have $A_{p}\left(y_{1}\right)=A\left(y_{1}\right) \in \operatorname{Lie} \operatorname{Aut}(K, \eta)$ and therefore $W:=\exp A\left(y_{1}\right) \in \operatorname{Aut}(K, \eta)$. So by definition we get

$$
\eta\left(x_{1}+x_{0}\right)=\eta\left(W\left(p+x_{0}\right)\right)=\eta(W e)[\eta(e)]^{-1} \eta\left(p+x_{0}\right)=\alpha \eta_{1}\left(x_{1}\right) \eta_{0}\left(x_{0}\right) \text {. }
$$

(b) For $\rho\left(x_{1 / 2}\right):=\eta\left(\exp A_{p}\left(x_{1 / 2}\right) e\right)$ one has $\rho\left(x_{1 / 2}+y_{1 / 2}\right)=$ $\alpha \rho\left(x_{1 / 2}\right) \rho\left(y_{1 / 2}\right)$ because of $\left[A_{p}\left(x_{1 / 2}\right), A_{p}\left(y_{1 / 2}\right)\right]=0$ (see [2, Satz 2.1]). Hence

$$
\begin{aligned}
\Delta_{x_{1 / 2}}^{y_{1 / 2}} \log \rho\left(x_{1 / 2}\right) & =\left.\frac{d}{d t}\right|_{0} \log \rho\left(x_{1 / 2}+t y_{1 / 2}\right)=\left.\frac{d}{d t}\right|_{0} \log \rho\left(t y_{1 / 2}\right) \\
& =-\sigma\left(h(e),\left.\frac{d}{d t}\right|_{0} \exp t A_{p}\left(y_{1 / 2}\right) e\right)=-\sigma\left(e, p y_{1 / 2}\right)=0
\end{aligned}
$$

by $h(e)=e$ and $A_{p}\left(y_{1 / 2}\right) e=p y_{1 / 2}$. We conclude $\eta\left(\exp A_{p}\left(x_{1 / 2}\right) e\right)=\eta(e)$ for all $x_{1 / 2} \in \mathfrak{A}_{1 / 2}$. Now (b) follows by Lemma 1.7 .

(c) We have to check (1.1) to (1.4). Obviously, (1.1) is satisfied. To prove (1.2) we remark that $A(p), A(e-p) \in \operatorname{Lie} \operatorname{Aut}(K, \eta)$ and get $\eta_{1}\left(\tau x_{1}\right)=$ $\eta_{1}\left(\exp \gamma A\left(p_{1}\right) x_{1}\right)=\left(\exp \gamma A\left(p_{1}\right)\left(x_{1}+p_{0}\right)\right)=\exp (\gamma r) \eta\left(x_{1}+p_{0}\right)=\tau^{r} \eta_{1}\left(x_{1}\right)$ for 
$\tau>0$; here we have used that the map $\alpha: \operatorname{Aut}(K, \eta) \rightarrow \mathbf{I R}^{+}, W \mapsto \alpha(W)$, which is defined by (1.5), is a continuous homomorphism of groups. The proof for $\eta_{0}$ is achieved in the same way.

The property (1.3) for $\eta_{i}$ immediately follows because $\eta$ satisfies (1.3). To verify (1.4) for $\eta_{0}$ let $x_{n}$ be a sequence of points of $K_{0}$ which converges to a boundary point of $K_{0}$. Then $p_{1}+x_{n}$ is a sequence in $K$ which converges to a boundary point of $K$ because of Corollary 1.10. Therefore $\eta_{0}\left(x_{0}\right)=\eta\left(p_{1}+\right.$ $x_{0}$ ) converges to $+\infty$. The proof for $\eta_{1}$ goes along the same lines.

We recall the remark of $\S 1$ that for (1.6) to (1.13) the property (1.5) of a triple $F$ is not used. We shall apply this to $F_{i}, i=0,1$.

LEMMA 2.2. For $i=0,1$ and all $v_{i}, w_{i} \in \mathfrak{A}_{i}, x_{i} \in K_{i}$ we have

(a) $\sigma_{F_{i}}\left(v_{i}, w_{i}\right)=\sigma_{F}\left(v_{i}, w_{i}\right)$

(b) $h_{F}\left(x_{1}+x_{0}\right)=h_{F_{1}}\left(x_{1}\right)+h_{F_{0}}\left(x_{0}\right)$,

(c) $\left.H_{F}\left(x_{1}+x_{0}\right)\right|_{\mathfrak{A}_{i}}=H_{F_{i}}\left(x_{i}\right)$,

(d) $\left.H_{F}\left(x_{1}+x_{0}\right)\right|_{\mathfrak{A}_{1 / 2}}=\left[A_{1 / 2}\left(x_{1}\right)\right]^{-1} A_{1 / 2}\left(h_{F_{0}}\left(x_{0}\right)\right)$,

(e) $\left.A_{F}\left(x_{i}\right)\right|_{\mathfrak{A}_{i}}=A_{F_{i}}\left(x_{i}\right)$.

Proof. (a) and (b) follow by direct calculation using Lemma 2.1. The part (e) is seen by differentiating (c). To prove (c) and (d), we have

$$
\begin{aligned}
H_{F}(x) w & =-\left.\frac{d}{d t}\right|_{0} h_{F}(x+t w) \\
& =-\left.\frac{d}{d t}\right|_{0}\left[\exp -A_{p}\left(k_{1 / 2}(x+t w)\right)\right]^{\sigma} h_{F}\left(x_{1}+t w_{1}+k_{0}(x+t w)\right)
\end{aligned}
$$

for all $x \in K, w \in V$. With $x=x_{1}+x_{0}$ we can easily derive (c) and (d).

Remark 2.3. (a) Instead of $h_{F_{i}}, H_{F_{i}}, A_{F_{i}}$ and $\sigma_{F_{i}}$, we write $h_{i}, H_{i}, A_{i}$ and $\sigma_{i}$, $i=0$, 1. Note that because of part (e) of Lemma 2.2 the definition of $A_{i}$, $i=0,1$, is unambiguous. Also for $i=1 / 2$ we put $\sigma_{1 / 2}\left(u_{1 / 2}, v_{1 / 2}\right):=\sigma\left(u_{1 / 2}\right.$, $\left.v_{1 / 2}\right)$. Whenever possible we drop the index of $\sigma$; most of the time it is determined by the index of the arguments.

(b) Instead of $K_{i}^{\sigma_{i}}$ we write $K_{i}^{\sigma}$ (for definition see $\S 1$ ).

The following result deals with the case $\mathfrak{A}_{F}=\mathfrak{S}_{F}$. It was contained (with a different proof) in a preliminary manuscript for [8].

For a Jordan-algebra we denote by $x^{-1}$ the inverse of an invertible element.

LEMMA 2.4. If $\mathfrak{A}=\widetilde{S}$ then

(a) $K$ is the positive cone of the formally-real Jordan-algebra $\mathfrak{A}$,

(b) $h(x)=x^{-1}, H(x)^{-1}=2 A(x)^{2}-A\left(x^{2}\right), x \in K$.

Proof. Part (a) is clear from Theorem 1.6. To prove (b) we have $H(x)^{-1}=$ $2 A(x)^{2}-A\left(x^{2}\right)=: P(x)$ because of $\mathfrak{A}=\subseteq$ from [5a, Satz 2.4]. But as $H(x) x$ $=h(x)$ by $[5 \mathrm{a},(1.6)]$ we get $h(x)=P(x)^{-1} x=x^{-1}$. 
For an arbitrary triple $F$ of $\mathscr{F}$ we get

Corollary 2.5. $h_{1}\left(x_{1}\right)=x_{1}^{-1}$ and $4 A_{1 / 2}\left(h_{1}\left(x_{1}\right)\right)=\left[A_{1 / 2}\left(x_{1}\right)\right]^{-1}$ for all $x_{1} \in$
$K_{1}$.

Proof. The first assertion is clear by Lemma 2.4. To prove the second one it suffices to note that the mapping $\mathfrak{X}_{1} \rightarrow\left(\text { End } \mathfrak{U}_{1 / 2}\right)^{+}, x_{1} \mapsto 2 A_{1 / 2}\left(x_{1}\right)$ is a homomorphism of Jordan-algebras by Theorem 1.2 and [2, Satz 2.1].

From Corollary 2.5, Lemma 2.2 and (1.10) it follows

COROllaRY 2.6. For $i=0,1$ and $x_{i} \in K_{i}^{\sigma}$ the endomorphism $A_{1 / 2}\left(x_{i}\right)$ is positive-definite (with respect to $\sigma$ ).

3. Splitting of Lie $\operatorname{Aut}(K, \eta)$.

1. Let $F=\langle K, \eta, e\rangle$ be a triple of $\mathscr{F}$ and $p \in \mathcal{S}, p^{2}=p$. We put

(3.1) $\searrow_{1}^{p}=$ Lie-subalgebra of Lie $\operatorname{Aut}(K, \eta)$ generated by $\left\{A\left(x_{1}\right) ; x_{1} \in \mathfrak{A}_{1}\right\}$,

(3.2) $\searrow_{1 / 2}^{p}:=\left\{A_{p}\left(x_{1 / 2}\right) ; x_{1 / 2} \in \mathfrak{A}_{1 / 2}\right\}$,

(3.3) $\searrow_{0}^{p}:=\left\{T \in \operatorname{Lie} \operatorname{Aut}(K, \eta) ; T \mathfrak{A}_{i} \subset \mathfrak{A}_{i}, i=0, \frac{1}{2}, 1, T \mathfrak{U}_{1}=0\right\}$.

(3.4) $\searrow^{p}=$ Lie-subalgebra of Lie Aut $(K, \eta)$ generated by $\delta_{1}^{p} \cup \delta_{1 / 2}^{p} \cup \delta_{0}^{p}$.

(3.5) $\delta_{i j}^{\mathrm{p}}:=\left\{\left.T\right|_{\mathfrak{A}_{j}} ; T \in \searrow_{i}^{p}\right\}, i=0,1, j=0, \frac{1}{2}, 1$.

(3.6) Let $i=0,1$ and $T_{i} \in \delta_{i}^{p}$ then $T_{i j}:=\left.T_{i}\right|_{\mathscr{A}_{j}}, j=0, \frac{1}{2}, 1$.

Finally we denote by $\Delta_{i}^{p}$ (resp. $\Delta_{i j}^{p}$, resp. $\Delta^{p}$ ) the connected Lie-subgroups of $\operatorname{Aut}(K, \eta)$ with Lie-algebra $\delta_{i}^{p}\left(\right.$ resp. $\searrow_{i j}^{p}$, resp. $\left.\searrow^{p}\right)$.

We drop the superscript " $p$ " if no confusion can arise. But there are several instances where we simultaneously have to use b's which are constructed with respect to different $p$ 's. We therefore cannot avoid indexing by $p$ at all.

2. In the remainder of this paragraph we are considering a triple $F=$ $\langle K, \eta, e\rangle$ of $\mathscr{F}$ and investigate the various $\delta$ 's which are formed with respect to a fixed $p \in S, p^{2}=p$. We may therefore drop the superscript $p$ in all statements. In the proofs, however, we are sometimes dealing with $b$ 's which are formed with respect to different idempotents of $\subseteq$. Then we are using the idempotents as superscript to avoid any confusion.

LEMMA 3.1. (a) There exists a homomorphism of Lie-algebras from $\delta_{11}$ to End $\mathfrak{A}_{1 / 2}$ which sends $A_{1}\left(x_{1}\right)$ to $A_{1 / 2}\left(x_{1}\right)$.

(b) $\mathrm{\delta}_{11}=A_{1}\left(\mathfrak{U}_{1}\right)+\operatorname{Der} \mathfrak{U}_{1}=A_{1}\left(\mathfrak{A}_{1}\right)+\left[A_{1}\left(\mathfrak{A}_{1}\right), A_{1}\left(\mathfrak{A}_{1}\right)\right]$,

(c) $\mathrm{d}=\mathrm{\delta}_{1}+\mathrm{\delta}_{1 / 2}+\grave{\delta}_{0}$ (direct sum of vectorspaces),

(d) $\delta_{1}=A_{p}\left(\mathfrak{A}_{1}\right)+\left[A_{p}\left(\mathfrak{A}_{1}\right), A_{p}\left(\mathfrak{A}_{1}\right)\right]$

(e) $\delta_{1 / 2}$ is an abelian ideal of $\delta$,

(f) $\delta_{0} \subset \operatorname{Der} \mathfrak{A}_{p}, \delta_{1 / 2} \subset$ Der $\mathfrak{A}_{p}$,

(g) For $i=0,1$ and all $T_{i} \in \delta_{i}, x_{1 / 2} \in \mathfrak{A}_{1 / 2}$ we have $\left[T_{i}, A_{p}\left(x_{1 / 2}\right)\right] \in \delta_{1 / 2}$ and $\left[T_{1}, T_{0}\right]=0$. 
Proof. Part (a) follows by [2, Lemma 2.3]. Part (b) is known from the theory of Jordan-algebras [1]. We get now (d) from (b) with (a). Furthermore, (f) is seen using Lemma 1.4(b), Theorem 1.2 and [2, Satz 2.1]. It only remains to prove parts (c), (e) and (g). To verify (g) we use (f) and (d) and get $\left[T_{1}, T_{0}\right]=0$ for all $T_{1} \in \searrow_{1}, T_{0} \in \searrow_{0}$. Because of (f) it remains only to prove $\left[T_{1}, A_{p}\left(x_{1 / 2}\right)\right] \in \mathrm{d}_{1 / 2}$. Here we may assume $T_{1}=A\left(x_{1}\right)$ and the assertion follows by (f). To prove (c) let $T_{1}+T_{1 / 2}+T_{0}=0, T_{i} \in \delta_{i}, i=0, \frac{1}{2}, 1$; then $T_{1} x_{1}=0, T_{1 / 2} x_{1}=0$ for all $x_{1} \in \mathfrak{A}_{1}$. So $T_{11}=0$ and $T_{1 / 2}=0$. By (a) we get $T_{1}=0$ and the sum $\delta_{1}+\delta_{1 / 2}+\delta_{0}$ is direct. It is also a Lie-algebra because of $(\mathrm{g})$. Hence (c) is proved. Finally, part (e) is clear because of $(\mathrm{g})$ and [9, IV, Theorem 6].

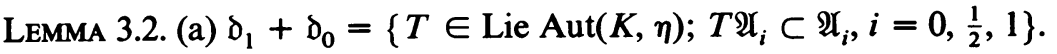

(b) $\searrow_{0}$ and $\searrow_{1}+\searrow_{0}$ are algebraic Lie-subalgebras of End $V$.

Proof. (a) Let $T \in \operatorname{Lie} \operatorname{Aut}(K, \eta)$ and $T \mathfrak{A}_{i} \subset \mathfrak{A}_{i}$ for $i=0, \frac{1}{2}, 1$. Then put $S:=\left.T\right|_{\mathscr{A}_{1}}$ and conclude $S \in \mathrm{D}_{11}$. So there exists $T_{1} \in \searrow_{1}$ with $T_{11}=S$ and $T-T_{1} \in \delta_{0}$ follows.

(b) By [3, I, Satz 1.8], there exists an algebraic group $\Gamma$ with Lie $\Gamma=$ Lie $\operatorname{Aut}(K, \eta)$. Put $\theta:=\left\{W \in \Gamma ; W \mathfrak{A}_{i} \subset \mathfrak{A}_{i}, i=0, \frac{1}{2}, 1\right\}$ and $\tilde{\theta}:=\{W \in \theta$; $\left.\left.W\right|_{\mathfrak{A}_{1}}=\mathrm{Id}\right\}$ and verify Lie $\theta=\delta_{1}+\delta_{0}$ and Lie $\tilde{\theta}=\delta_{0}$.

3. In this section we characterize the case $\delta=\operatorname{Lie} \operatorname{Aut}(K, \eta)$. For this we first note that a $T \in \operatorname{Lie} \operatorname{Aut}(K, \eta)$ can be written at most in one way as $T=A_{p}\left(x_{1}\right)+A_{p}\left(x_{1 / 2}\right)+D$ with $x_{i} \in \mathfrak{A}_{i}$ and $D \in \operatorname{Der} \mathfrak{A}_{p}, D p=0$.

THEOREM 3.3. The following statements are equivalent:

(a) $\mathfrak{A}_{1}$ is an ideal of $\mathfrak{S}$,

(b) $T^{o} p \in \mathfrak{A}_{1}$ for all $T \in \operatorname{Lie} \operatorname{Aut}(K, \eta)$,

(c) Lie $\operatorname{Aut}(K, \eta) \subset A_{p}\left(\mathfrak{A}_{1}\right)+\operatorname{Der} \mathfrak{A}_{p}$,

(d) Every $T \in \operatorname{Lie} \operatorname{Aut}(K, \eta)$ can be written in a unique way $T=A_{p}\left(x_{1}\right)+$ $A_{p}\left(x_{1 / 2}\right)+D$ with $x_{i} \in \mathfrak{A}_{i}, i=1, \frac{1}{2}$, and $D \in \operatorname{Der} \mathfrak{U}_{p}, D p=0$,

(e) Lie $\operatorname{Aut}(K, \eta)=\delta$.

(f) $\left\{x_{1 / 2} \in \mathfrak{U}_{1 / 2} ; A_{p_{0}}\left(x_{1 / 2}\right) \in \operatorname{Lie} \operatorname{Aut}(K, \eta)\right\}=\{0\}$.

Proof. We first prove (c) $\Rightarrow(\mathrm{d})$. Let $T \in \operatorname{Lie} \operatorname{Aut}(K, \eta)$ and write $T=$ $A_{p}\left(x_{1}\right)+D$ by (c). Obviously $D p \in \mathfrak{A}_{1 / 2}$ and we get $T=A_{p}\left(x_{1}\right)+A_{p}(2 D p)$ $+D^{\prime}, D^{\prime} \in \operatorname{Lie} \operatorname{Aut}(K, \eta) \cap \operatorname{Der} \mathfrak{A}_{p}, D^{\prime} p=0$. Using the remark preceding the theorem we can now prove (d). To see (d) $\Rightarrow(e)$ it is sufficient to show that $D \in \operatorname{Lie} \operatorname{Aut}(K, \eta) \cap \operatorname{Der} \mathfrak{A}_{p}, D p=0$ implies $D \in \delta$. From the premises we get $D \mathfrak{A}_{i} \subset \mathfrak{A}_{i}$ for $i=1, \frac{1}{2}, 0$. Hence $\hat{D}:=\left.D\right|_{\mathfrak{A}_{1}} \in$ Der $\mathfrak{A}_{1}$. We know from Jordan-theory, [1, IX, Satz 3.1], that all derivatives of the formally-real Jordan-algebra $\mathfrak{A}_{1}$ are "inner", i.e. we have $\hat{D}=\Sigma_{(x, y) \in R}\left[A_{1}(x), A_{1}(y)\right]$ with 
appropriate $R \subset \mathfrak{A}_{1} \times \mathfrak{A}_{1}$. Put $D_{1}:=\Sigma_{(x, y) \in R}[A(x), A(y)]$ and $D_{0}:=D-$ $D_{1}$ and (e) follows.

To prove $(\mathrm{e}) \Rightarrow(\mathrm{f})$, let $A_{p_{0}}\left(x_{1 / 2}\right) \in \operatorname{Lie} \operatorname{Aut}(K, \eta)$ and write $A_{p_{0}}\left(x_{1 / 2}\right)=T_{1}$ $+T_{1 / 2}+T_{0}$ with $T_{i} \in \mathfrak{d}_{i}$ by (e). Applying both sides to arbitrary $x_{1} \in \mathfrak{A}_{1}$, we get $T_{1}=0, T_{1 / 2}=0$. But then $\frac{1}{2} x_{1 / 2}=A_{p_{0}}\left(x_{1 / 2}\right) p_{0}=T_{0} p_{0} \in \mathfrak{A}_{0} \cap \mathfrak{A}_{1 / 2}$ $=0$. For $(\mathrm{f}) \Rightarrow$ (a) we put $d_{1}:=p, d_{2}:=c-p, d_{3}:=e-c$ and form the Peirce-decomposition of $\mathfrak{A}$ with respect to $d_{1}, d_{2}, d_{3}$. The assertion (a) is now equivalent to $\mathfrak{A}_{12}=0$. But, for $x \in \mathfrak{A}_{12}$, we have $x \in \mathfrak{U}_{1 / 2}(p)$ and $A_{d_{2}}(x)=$

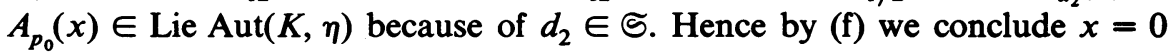
and the assertion (a) follows. To see (a) $\Rightarrow$ (b) we first remark that because of Lemma 1.4. the conclusion (c) $\Rightarrow(\mathrm{d}) \Rightarrow(\mathrm{e})$ is always true for $p:=c$. Hence for every $T \in \operatorname{Lie} \operatorname{Aut}(K, \eta)$ we get $T=T_{1}+A_{c}\left(x_{1 / 2}\right)+T_{0}$ where now $T_{i} \in \delta_{i}^{c}$ and $x_{1 / 2} \in \mathfrak{A}_{1 / 2}(c)$. But $T_{0}^{\sigma} \mathfrak{A}_{1}(c)=0$ and $\left[A_{c}\left(x_{1 / 2}\right)\right]^{\sigma} \mathfrak{A}_{1}(c)=$ $A_{c_{0}}\left(x_{1 / 2}\right) \mathfrak{A}_{1}(c)=0$ [2]. Because of $p \in \mathfrak{A}_{1}(c)=\subseteq$ it now suffices to quote Lemma 3.1(d) and $A_{c}\left(x_{1}\right)=A\left(x_{1}\right)$ for $x_{1} \in \mathfrak{X}_{1}(c)$. Finally, to prove (b) $\Rightarrow$ (c) for $T \in \operatorname{Lie} \operatorname{Aut}(K, \eta)$ we put $\hat{T}:=T-A_{p}\left(x_{1}\right)-A_{p}\left(2 x_{1 / 2}\right)$ where $x_{i} \in \mathfrak{A}_{i}$ and $T p=x_{1}+x_{1 / 2}+x_{0}$. Then we have $\hat{T}_{p} \in \mathfrak{A}_{0}$. Further, in Theorem 1.2(a) we put $b=p, v=p_{0}$ and use (b); so we get $\hat{T}_{p_{0}} \in \mathfrak{A}_{0}$. Because of $\hat{T}+\hat{T}^{\sigma}=2 A(\hat{T} e)[4,(I ; 4.2)]$, we conclude $\hat{T}^{\sigma} p=2 p\left(\hat{T} p+\hat{T} p_{0}\right)-\hat{T} p \in \mathfrak{U}_{0}$; but by hypothesis $\hat{T}^{\sigma} p \in \mathfrak{A}_{1}$. So $\hat{T}^{\sigma} p=0$ and $\hat{T} \in \operatorname{Der} \mathfrak{A}_{p}$ by Lemma 1.4 .

Last but not least we note that in exploiting the identity $\sigma(h(x), T x)=$ $\sigma(e, T e)$ of [4, I, Lemma 1.1], in a straightforward calculation we get

THEOREM 3.4. For $x_{1 / 2} \in \mathfrak{A}_{1 / 2}$ the following are equivalent;

(a) $A_{p_{0}}\left(x_{1 / 2}\right) \in \operatorname{Lie} \operatorname{Aut}(K, \eta)$,

(b) (i) $\frac{1}{4} x_{1 / 2}=h_{0}\left(y_{0}\right) \cdot x_{1 / 2} y_{0}$ for all $y_{0} \in K_{0}$,

(ii) $p_{0}\left\{y_{1 / 2} \cdot y_{1 / 2}\left[p_{1}\left(x_{1 / 2} \cdot y_{1} y_{1 / 2}\right)\right]\right\}=p_{0}\left\{y_{1 / 2} \cdot x_{1 / 2}\left[p_{0}\left(y_{1 / 2} \cdot y_{1} y_{1 / 2}\right)\right]\right\}$ for all $y_{1} \in \mathfrak{A}_{1}$ and all $y_{1 / 2} \in \mathfrak{U}_{1 / 2}$.

REMARK 3.5. It is remarkable that by the above theorem one can express part (f) of Theorem 3.3 without using Lie $\operatorname{Aut}(K, \eta)$. We shall take advantage of this fact later on.

4. In this section, we investigate the homogeneity properties of the cones $K_{i}$.

LEMMA 3.6. Let $i=0,1$ then

(a) $\Delta_{i i} \subset \operatorname{Aut}\left(K_{i}, \eta_{i}\right)$,

(b) $\Delta_{11}$ operates transitively on $K_{1}$,

(c) $A_{1 / 2}\left(T_{i i}^{\sigma} x_{i}\right)=T_{i 1 / 2}^{\sigma} A_{1 / 2}\left(x_{i}\right)+A_{1 / 2}\left(x_{i}\right) T_{i 1 / 2}$ for all $T_{i} \in \delta_{i}, x_{i} \in \mathfrak{A}_{i}$.

Proof. Part (a) is immediate and (b) is known from Jordan-theory [1, XI, Satz 2.4]. Finally (c) comes out by (1.13) and Lemma 2.2(d).

The following theorem will often be used. It is particularly important for the main results of this paper. 
THEOREM 3.7. (a) $\Delta$ operates transitively on $K$,

(b) $\Delta_{00}$ operates transitively on $K_{0}$.

Proof. For (a), by Theorem 1.8., it suffices to show that $\Delta_{11} \times \Delta_{00}$ operates transitively on $K_{1}+K_{0}$. So (a) follows from (b). To prove (b) we first assume Lie $\operatorname{Aut}(K, \eta)=\delta=\mathfrak{d}^{p}$. Now $\operatorname{Aut}(K, \eta)$ is transitive on $K$, hence Lie $\operatorname{Aut}(K, \eta) \rightarrow V, T \mapsto T x, x \in K$, is a surjective map. Because of $\delta_{i}\left(x_{1}+\right.$ $\left.x_{0}\right) \subset \mathfrak{A}_{i}$ for $x_{1}+x_{0} \in K_{1}+K_{0}$ we get a surjective map $\searrow_{00} \rightarrow \mathfrak{U}_{0}, T_{00} \mapsto$ $T_{00} x_{0}, x_{0} \in K_{0}$. So the orbit of $\Delta_{00}$ through $x_{0} \in K_{0}$ is open in $\mathfrak{U}_{0}$. But $K_{0}$ is connected and the assertion follows.

In the general case we form the Peirce-decomposition of $\mathfrak{A}$ with respect to the CSI $d_{1}, d_{2}, d_{3}$, defined by $d_{1}:=p, d_{2}:=c-p, d_{3}:=e-c$. One easily gets

(1) $\mathfrak{A}_{1}=\mathfrak{A}_{11}, \mathfrak{A}_{1 / 2}=\mathfrak{A}_{13}+\mathfrak{A}_{12}, \mathfrak{A}_{0}=\mathfrak{A}_{33}+\mathfrak{A}_{23}+\mathfrak{A}_{22}$.

(2) $\mathfrak{A}_{22}$ is a formally-real Jordan-subalgebra of $\mathfrak{S}$.

(3) $D_{0}$ contains the Lie-subalgebra $D^{\prime}$ of $\operatorname{Lie} \operatorname{Aut}(K, \eta)$ that is generated by $\delta_{0}^{c} \cup A\left(\mathfrak{A}_{22}\right) \cup A_{d_{2}}\left(\mathfrak{A}_{23}\right)$. Further we use Theorem 1.8 for $c$ and Corollary 1.9 for $p$ and get

(4) $K_{0}=K_{0}^{p}=\cup_{x_{23} \in \mathscr{R}_{23}} \exp A_{d_{2}}\left(x_{23}\right)\left(K_{22}+K_{33}\right)$.

Here $K_{i i}$ denotes the image of $K$ under the orthogonal projection of $\mathfrak{A}$ onto $\mathfrak{A}_{i i}$. Obviously $K_{33}=K_{0}^{c}$, so, by what we have proved above, the group $\Delta_{33}$ generated by $\left.\delta_{0}^{p}\right|_{\mathfrak{A}_{33}}$ operates transitively on $K_{33}$. The group $\Delta_{22}$ generated by $A\left(\mathfrak{A}_{22}\right)$ operates transitively on $K_{22}$ because $K_{22}$ is the positive cone of the formally-real Jordan-algebra $\mathfrak{U}_{22}$. Hence by (3) and (4) the subgroup $\Delta_{00}^{\prime}$ of $\Delta_{00}^{p}$ generated by $\left.\delta^{\prime}\right|_{\mathscr{A}_{0}}$ operates transitively on $K_{0}$.

COROLlaRY 3.8. $F_{1}$ and $F_{0}$ lie in $\mathscr{F}$ for all $p \in \mathfrak{S}_{F}, p^{2}=p$.

It is well known that for a homogeneous cone $K$ "the invariant" $\iota(K ; x)$ of $K$, which is given by $\iota(K ; x):=\int_{K^{\circ}} \exp (-\sigma(x, y)) d y$, satisfies (1.1) to (1.5) [5]. Furthermore, $\left.\iota(K ; x)^{2}=\alpha \operatorname{det} H_{F}(x), \alpha\right\rangle 0$, for all triples $F=\langle K, \eta, e\rangle$ of $\mathscr{F}$ [5]. Using Lemma 2.2 we get

Corollary 3.9. (a) $\iota(K ; x)=\imath\left(K ; x_{1}+k_{0}(x)\right)$ for all $x \in K$.

(b) $\iota\left(K ; x_{1}+x_{0}\right)=\alpha \iota\left(K_{1} ; x_{1}\right) \iota\left(K_{0} ; x_{0}\right)\left[\operatorname{det} A_{1 / 2}\left(h_{0}\left(x_{0}\right)\right) A_{1 / 2}\left(h_{1}\left(x_{1}\right)\right)\right]^{1 / 2}$ for all $x_{1} \in K_{1}, x_{0} \in K_{0}$. $K_{1}$.

(c) $\iota_{1}\left(K ; x_{1}\right)=\iota\left(K ; x_{1}+p_{0}\right)=\alpha_{1} \iota\left(K_{1} ; x_{1}\right)\left[\operatorname{det} A_{1 / 2}\left(h_{1}\left(x_{1}\right)\right)\right]^{1 / 2}$ for all $x_{1} \in$

(d) $\iota_{0}\left(K ; x_{0}\right)=\iota\left(K ; p_{1}+x_{0}\right)=\alpha_{0} \iota\left(K_{0} ; x_{0}\right)\left[\operatorname{det} A_{1 / 2}\left(h_{0}\left(x_{0}\right)\right)\right]^{1 / 2}$ for all $x_{0} \in$ $K_{0}$.

Note that we are still dealing with a fixed triple $F$ of $\mathscr{F}$ and a fixed idempotent $p \in \subseteq=\subseteq_{F}$. All expressions of Corollary 3.9 are formed with respect to that idempotent. 


\section{On sums of cones and the self-dual case.}

1. In this subsection we are dealing with several triples of $\mathscr{F}$. We therefore of ten use the triple designator as a subscript.

THeOREM 4.1. Let $F=\langle K, \eta, e\rangle$ be a triple of $\mathscr{F}$. Let $V_{i}, i=1, \ldots, r$, be vector subspaces of $V=V_{F}$ such that $V=\bigoplus_{i=1}^{r} V_{i}$. Further let $Z_{i}$ be the image of $K$ under the canonical projection of $V$ onto $V_{i}$. Then the following are equivalent.

(a)(i) The spaces $V_{i}$ are pairwise orthogonal with respect to $\sigma_{F}$,

(ii) $K=\bigoplus_{i=1}^{r} Z_{i}:=\left\{\sum_{i=1}^{r} z_{i} ; z_{i} \in Z_{i}\right\}$.

(b) The spaces $V_{i}$ are ideals of $\mathfrak{A}_{F}$.

Proof. (a) $\Rightarrow$ (b) Using the hypothesis (ii), we have the well-known identity, Lie $\operatorname{Aut}(K, \iota(K ;-))=\bigoplus_{i=1}^{r} \operatorname{Lie} \operatorname{Aut}\left(Z_{i}, \iota\left(Z_{i} ;-\right)\right)$. Hence $H(x)=$ $H_{F}(x)$ leaves all $V_{i}$ invariant because of (1.5), (i) and (1.13). Now differentiation of $H(x)$ at $x=e=e_{F}$ gives the desired result.

(b) $\Rightarrow$ (a) First we easily derive (i) by (1.11) from (b). Now we prove the assertion by induction on $n=\operatorname{dim} \mathfrak{A}, \mathfrak{A}=\mathfrak{A}_{F}$. The case $n=1$ being trivial we have to conclude from $n-1$ to $n$. We distinguish two cases:

1. $\mathfrak{A}=\mathfrak{S}$. Here $K$ is by Theorem 1.6 the positive cone of the formally-real Jordan-algebra $\mathfrak{A}$ and the assertion follows from Jordan-theory [7, VI, §7].

2. $\mathfrak{A} \neq \mathfrak{S}$. Put $c=: \sum_{i=1}^{r} d_{i}, d_{i} \in V_{i}$; then the Peirce-decomposition $\mathfrak{A}=$ $\mathfrak{A}_{1}(c)+\mathfrak{A}_{1 / 2}(c)+\mathfrak{A}_{0}(c)$ splits into a sum of Peirce-decompositions of the $V_{i}$. With $V_{i j}:=\left\{x_{i} \in V_{i} ; d_{i} x_{i}=j x_{i}\right\}, j=0, \frac{1}{2}, 1$, we get $\mathfrak{A}_{j}(c)=\bigoplus_{i=1}^{r} V_{i j}$. It is clear that for $j=0,1$ the $V_{i j}$ are ideals of $\mathfrak{A}_{j}(c)=\mathfrak{A}_{F_{j}}$. Therefore, by induction, we conclude that $K_{j}, j=0,1$, is the direct sum of its projections on $V_{i j}$. By Theorem 1.8 the desired result now follows.

THEOREM 4.2. Under the assumptions of Theorem 4.1 let (a) or (b) of Theorem 4.1 be satisfied. Put $e=: \sum_{i=1}^{r} d_{i}, d_{i} \in V_{i}$ and $\eta_{i} ; Z_{i} \rightarrow \mathbf{R}^{+}$, $\eta_{i}\left(z_{i}\right):=\eta\left(z_{i}+\sum_{j \neq i} d_{j}\right)$ then

(a) $R_{i}:=\left\langle Z_{i}, \eta_{i}, c_{i}\right\rangle$ is a triple of $\mathscr{F}$.

(b) $\eta\left(\sum_{i=1}^{r} z_{i}\right)=\alpha \Pi_{i=1}^{r} \eta_{i}\left(z_{i}\right), \alpha>0$, for all $z_{i} \in Z_{i}, i=1, \ldots, r$.

(c) $\operatorname{Aut}(K, \eta)_{0}=\Pi_{i=1}^{r} \operatorname{Aut}\left(Z_{i}, \eta_{i}\right)_{0}$ (connected components).

(d) $\mathfrak{S}_{F}=\bigoplus_{i=1}^{r} \mathfrak{S}_{R_{i}}$.

Proof. As in the first part of the proof of Theorem 4.1, we get $H(x) V_{i} \subset$ $V_{i}$. We put $H(x ; v):=\Delta_{x}^{v} H(x)$ and get $H\left(x ; v_{i}\right) v_{j}=H\left(x ; v_{j}\right) v_{i} \in V_{i} \cap V_{j}$. So $H(x) v_{i}$ only depends on $x_{i}$ and $v_{i}$. We put $N_{i}\left(x_{i}\right):=\left.H(x)\right|_{v_{i}}$ where $x \in K$ such that the $i$ th component coincides with $x_{i}$. Now, we define $a_{i}:=\left\{T_{i} \in\right.$ End $V_{i} ; N_{i}\left(x_{i} ; T_{i} x_{i}\right)+T_{i}^{\sigma} N_{i}\left(x_{i}\right)+N_{i}\left(x_{i}\right) T=0$ for all $\left.x_{i} \in Z_{i}\right\}$ and easily get Lie $\operatorname{Aut}(K, \eta)=\bigoplus_{i=1}^{r} a_{i}$ by using [4, I, Satz 1.8 and I; Lemma 1.1]. As an immediate consequence of this fact the group $\Omega_{i}$ generated by $a_{i}$ operates 
transitively on $Z_{i}$. Now the assertions of the theorem follow by simple calculations.

A triple $F$ of $\mathscr{F}$ is called reducible, if there exist (nontrivial) triples $R_{i}=\left\langle Z_{i}, \eta_{i}, d_{i}\right\rangle$ of $\mathscr{F}$ such that $K=\bigoplus Z_{i}$ and $\eta_{F}\left(\sum z_{i}\right)=\alpha \Pi \eta_{i}\left(z_{i}\right)$ for some $\alpha>0$ and all $z_{i} \in Z_{i}$.

COROLlary 4.3. A triple $F$ of $\mathscr{F}$ is reducible if and only if $\mathfrak{A}_{F}$ has nontrivial ideals.

We recall that we can construct by [5a, §1] from a triple $F=\langle K, \eta, e\rangle$ of $\mathscr{F}$ the triple $\tilde{F}=\left\langle K^{\sigma}, \tilde{\eta}, e\right\rangle$ of $\mathcal{F}$ where $\tilde{\eta}(y)=\left[\eta\left(h^{-1}(y)\right)\right]^{-1}$.

COROLLARY 4.4. The following are equivalent;

(a) $\mathfrak{S}_{F} \cap \mathfrak{S}_{\tilde{F}} \neq 0$,

(b) there exists an ideal $0 \neq \mathfrak{Q}$ of $\mathfrak{A}_{F}$ with $\mathfrak{Q} \subset \mathfrak{S}_{F}$,

(c) either $\mathfrak{A}_{F}$ is a formally-real Jordan-algebra and $K_{F}$ its positive cone, or $F$ is reducible and at least one of the summands $Z_{i}$ of $K_{F}$ is self-dual with respect to $\sigma_{R i}$ and at least one is not.

Proof. (a) $\Rightarrow$ (b) Let $d$ be the unit of the formally-real Jordan-algebra $\mathfrak{T}:=\mathfrak{S}_{F} \cap \mathfrak{S}_{\tilde{F}}$. Then, by Theorem 1.2 , we get $A_{x}(a) d \in \mathfrak{I}$ for all $a \in \mathfrak{A}$, $x \in \mathfrak{T}$. From this we conclude $x a \in \mathfrak{I}$ for all $x \in \mathfrak{T}, a \in \mathfrak{A}$.

(b) $\Rightarrow$ (c) Let $\mathfrak{Q}$ be a maximal ideal of $\mathfrak{A}=\mathfrak{A}_{F}$ that satisfies (b). Then either $\mathfrak{Q}=\mathfrak{A}$ and the first assertion follows by Theorem 1.5 or $0 \neq \mathfrak{Q} \neq \mathfrak{A}$. In this case $\mathscr{Q}$ is the sum of some $\widetilde{S}_{R_{i}}$ (see Theorem 4.2) and so at least one $Z_{i}$ is self-dual with respect to $\tau_{i}, \tau:=\sigma_{R_{i}}$, by Theorem 1.6. If $Z_{i}^{\tau_{i}}=Z_{i}$ for all $i$ then $K^{\sigma}=K$ and $\mathfrak{Q}=\mathfrak{A}$ by Theorem 4.5 .

(c) $\Rightarrow$ (a) From the hypothesis we easily deduce $Z_{i}^{\tau_{i}}=Z_{i}$ for at least one $i$ so $\mathfrak{A}_{R_{i}}=\widetilde{S}_{R_{i}}$ for at least one $i$ by Theorem 4.5. But $\mathfrak{S}_{R_{i}}=\mathfrak{S}_{\tilde{R}_{i}}$ for such $i$ and the assertion follows.

2. In the remainder of this paragraph, we may again consider a fixed triple $F=\langle K, \eta, e\rangle$ of $\mathscr{F}$.

THEOREM 4.5. Assume $K^{\sigma}=K$. Then

(a) $\mathfrak{A}=\mathfrak{S}$.

(b) If $\mathfrak{A}$ is simple, then $\eta(x)=\alpha \iota(K ; x)^{\beta}, \alpha, \beta>0$, for all $x \in K$.

Proof. Because of Theorem 4.2 we may assume $\mathfrak{A}$ is simple. Now, for $K, \sigma$ and $\iota=\iota(K ;-)$, we form the triple $G:=\langle K, \iota, d\rangle$, where $d \in K$ is the unique fixed point of $h_{\iota, \sigma}$ (see [5, Satz 4.4]). For simplicity of notation we put $\mathfrak{\Im}:=\mathfrak{U}_{G}$ and denote the left-multiplications in $\mathfrak{\Im}$ by $J(x)$. From the results of Chapters II and VI of [7] we get that $\mathfrak{s}$ is a formally-real Jordan-algebra with unit $d, K$ is the positive cone of $\mathfrak{\Im}$ and $J(x)=J(x)^{\sigma}$ for $x \in \mathfrak{\Im}$. Putting $\tau(u, v):=$ Trace $J(J(u) v)$ we have moreover $\sigma=\sigma_{G}=\tau$. From this we conclude that $\mathfrak{\Im}$ is simple, for otherwise $\mathfrak{\Im}$ would split into a sum of subspaces $V_{i}$ 
which are orthogonal with respect to $\tau=\sigma$ and $K$ would be the sum of its projections onto $V_{i}$. So, by Theorem 4.1, the algebra $\mathfrak{A}$ would not be simple, a contradiction.

Furthermore by $[5 \mathrm{a},(1.17)]$ we have $2 A(T e)=T+T^{\circ}=T+T^{\sigma_{G}}=$ $2 J(T d)$. We choose an injective map $v \mapsto T(v)$ of $V$ into Lie $\operatorname{Aut}(K, \eta)$ such that $T(e)=$ Id and $T(v) e=v, v \in V$. Such a map exists by [4, I, §3]. We define $R: V \rightarrow V$ by $R v:=T(v) d$. Then we get $u v=A(u) v=J(T(u) d) v=$ $J(R u) v$. By the commutativity of $\mathfrak{A}$ and $\mathfrak{s}$ we conclude $J(R u)=J(u) R$ and hence $J(R d)=R$. Since $J(x)$ and $R$ are selfadjoint with respect to $\sigma$ we derive $[J(u), R]=0$ from $J(R u)=J(u) R$. As $\mathfrak{\Im}$ is simple, $R=\delta$ Id, $\delta \neq 0$. So we get $\sigma(e, T e)=\delta^{-2} \sigma(d, T d)=\delta^{-2}$ Trace $J(T d)=\delta^{-2}$ Trace $A(T e)$ and, with $\beta:=\delta^{-2}$ and $[4, \S 1]$, it follows that $\eta(\exp T x)=$ $\exp (-\beta$ Trace $A(T e)) \eta(x)=(\operatorname{det} \exp T)^{-\beta} \eta(x)$ for all $x \in K, T \in$ Lie $\operatorname{Aut}(K, \eta)$. Therefore $\eta(x)=\alpha \iota(x)^{\beta}$ and the theorem is proved.

Theorem 4.6. Assume that $\mathfrak{A}$ is a Jordan-algebra. Then $\mathfrak{A}=\mathfrak{S}$.

Proof. (a). We may again assume that $\mathfrak{A}$ is simple. Hence we get a $\beta \in \mathbf{R}$ such that $\sigma(v, w)=\beta$ Trace $A(v w)$ for all $v, w \in V$. For $T \in \operatorname{Lie} \operatorname{Aut}(K, \eta)$ we conclude $\eta(\exp T x)=\exp (-\sigma(e, T e)) \eta(x)=\exp (-\beta$ Trace $T) \eta(x)=$ $[\text { det } \exp T]^{-\beta} \eta(x)$ for $x \in K$, such that $\eta(x)=\alpha u(K ; x)^{\beta}, x \in K$.

(b). Now, let $Z$ be the positive cone of $\mathfrak{A}$ and $G:=\langle Z, \iota(Z ;-), e\rangle$. Then $G$ is a triple of $\mathscr{F}$ and by [6, VI, Theorems 1, 2] we know that $H_{G}(x)=$ $P_{\mathfrak{A}}(x)^{-1} \in \operatorname{Aut}\left(K_{G}, \eta_{G}\right)$. We differentiate and get $\mathfrak{A}_{G}=\mathfrak{A}$ and $A(x) \in$ Lie $\operatorname{Aut}\left(K_{G}, \eta_{G}\right)$ for all $x \in \mathfrak{A}$. This implies $\mathfrak{A}=\mathfrak{S}_{G}$. For the unit $c$ of $\mathfrak{S}$, we have $c \in \subseteq_{G}$. From Theorem 1.8 it follows that $Z=\cup_{x_{1 / 2} \in \mathfrak{A}_{1 / 2}(c)}$ $\exp A_{c}\left(x_{1 / 2}\right)\left(Z_{1}+Z_{0}\right)$, where now $Z_{i} \subset \mathfrak{A}_{i}(c)$ are the positive cones of $\mathfrak{A}_{i}(c)$.

(c). We are going to prove $K=Z$ by induction on $n=\operatorname{dim} \mathfrak{A}$. The case $n=1$ is trivial. Hence we may assume $n>1$. This case $\mathfrak{A}=\subseteq$ being settled by Theorem 1.6, we assume $\mathfrak{A} \neq \subseteq$. Here we know that $F_{0}$ is a triple of $\mathscr{F}$ by Corollary 3.8. Further, $\mathfrak{A}_{F_{0}}=\mathfrak{A}_{0}(c)$ by Lemma 2.2. Hence $\mathfrak{A}_{F_{0}}$ is a Jordan-algebra and because of $\operatorname{dim} \mathfrak{A}_{0}(c)<\operatorname{dim} \mathfrak{A}$ we may apply the induction hypothesis to get $\mathfrak{A}_{F_{0}}=\mathfrak{S}_{F_{0}}$. This implies $Z_{0}=K_{0}^{c}$ by Theorem 1.6. By the same theorem we always have $Z_{1}=K_{1}^{c}$. We use Theorem 1.8 and the description of $Z$ by (b) to conclude $Z=K$. Hence (a) gives $\eta(x)=\alpha \iota(Z ; x)^{\beta}$. From this we obviously get $\mathfrak{A}=\mathfrak{A}_{G}$. But this implies $A(x) \in \operatorname{Lie} \operatorname{Aut}(K, \eta)$ for all $x \in \mathfrak{A}$ and the assertion follows.

Finally, we sum up.

THEOREM 4.7. The following conditions are equivalent:

(a) $\mathfrak{A}$ is Jordan-algebra,

(b) $K$ is self-dual with respect to $\sigma$,

(c) $\mathfrak{A}=\mathfrak{S}$. 


\section{Connection with left-symmetric algebras.}

1. We note that a Lie-subalgebra $g$ of endomorphisms of some finite dimensional vector space $L$ over $\mathbf{R}$ is called trigonalizable if and only if there exists a basis of $L$ such that all elements of $g$ are upper triangular matrices with respect to this basis. An analogous definition for groups of endomorphisms holds [13], [5a]. Now let $F$ be a triple of $\mathscr{F}$ and $\Gamma$ a connected, trigonalizable subgroup of $\operatorname{Aut}(K, \eta)$ that operates transitively on $K$ (in what follows we shall call such a group a ctt-subgroup of $\operatorname{Aut}(K, \eta)$ and its Lie-algebra a ctt-algebra for $F$ ). Then it is well known that $\Gamma$ is closed in $\operatorname{Aut}(K, \eta)$ and Lie $\Gamma \rightarrow V, T \mapsto T x$ is, for all $x \in K$, an isomorphism of vectorspaces [13], [5a]. For $x=e=e_{F}$ we write the inverse mapping $V \rightarrow$ Lie $\Gamma$ as $v \mapsto T(v)$ and so have $T(e)=\operatorname{Id}$ and $T(v) e=v, v \in V$. It is known, [5a], [13], that $(u, v) \mapsto T(u) v$ defines a left-symmetric algebra on $V$.

2. We fix again a triple $F$ of $\mathcal{F}$ and describe the ctt-algebras for $F$ in terms of $\mathfrak{A}_{F}$, thus connecting $\grave{\mathrm{E}}$. Vinberg's "left-symmetric" algebras with $\mathbf{M}$. Koecher's commutative algebra $\mathfrak{A}=\mathfrak{U}_{F}$. Note that $\mathfrak{X}$ was defined just before Remark 1.3.

THEOREM 5.1. Let a be a ctt-algebra for F. Then there exists a CSI $d_{1}, \ldots, d_{r}$ of idempotents $d_{i} \in \mathfrak{X}$ such that in terms of the Peirce-decomposition $\mathfrak{U}=\bigoplus_{1<i<j<r} \mathfrak{A}_{i j}$ of $\mathfrak{A}$ with respect to $d_{1}, \ldots, d_{r}$ there holds

(a) $T\left(d_{i}\right) d_{i}=d_{i} \neq 0$ for all $i=1, \ldots, r$,

(b) $\mathfrak{A}_{i i}=\mathbf{R} d_{i}$ for all $i=1, \ldots, r$,

(c) $d_{1} \in \mathfrak{S}_{F}$,

(d) $T\left(x_{i i}\right)=A\left(x_{i i}\right)$ for all $1 \leqslant i \leqslant r, x_{i i} \in \mathfrak{U}_{i i}$,

(e) $T\left(x_{i j}\right)=2 A_{d_{i}}\left(x_{i j}\right)$ for all $1 \leqslant i<j \leqslant r, x_{i j} \in \mathfrak{U}_{i j}$.

Proof. (1) The proof proceeds by induction on $n=\operatorname{dim} \mathfrak{A}$. The case $n=1$ is trivial. Choose now $0 \neq d_{1} \in \mathfrak{S}, d_{1}^{2}=d_{1}$, as in [5a, Satz 5.2] and form the Peirce-decomposition $\mathfrak{A}=\mathfrak{A}_{1}+\mathfrak{A}_{1 / 2}=\mathfrak{A}_{0}$ of $\mathfrak{A}$ with respect to $d_{1}$. Put $\mathfrak{a}_{00}:=\left\{\left.T\left(x_{0}\right)\right|_{\mathfrak{A}_{0}} ; x_{0} \in \mathfrak{U}_{0}\right\}$; then it is easy to see that $\mathfrak{a}_{00}$ is the Lie-algebra of a ctt-subgroup of $\operatorname{Aut}\left(K_{0}^{d_{1}}, \eta_{0}\right)$. So by induction we get $d_{2}, \ldots, d_{r} \in \mathfrak{A}_{0}$ satisfying (a) to (e) for $F_{0}$.

(2) For $T:=T\left(d_{i}\right), 1 \leqslant i \leqslant r$, we have $T \mathfrak{A}_{j} \subset \mathfrak{A}_{j}, j=0, \frac{1}{2}, 1, T \mathfrak{A}_{1}=0$ and $(T e)^{2}=T^{2} e=T e$. Further $T$ has only real eigenvalues. Now, using $T+T^{o}$ $=2 A(T e)$ and Lemma 3.6 we get $A_{1 / 2}(T e)=A_{1 / 2}\left(T^{\sigma} T e\right)=\hat{T}^{\sigma} A_{1 / 2}(T e)+$ $A_{1 / 2}(T e) \hat{T}=2\left[A_{1 / 2}(T e)\right]^{2}+\left[A_{1 / 2}(T e), \hat{T}\right], \hat{T}:=\left.T\right|_{\mathscr{R}_{1 / 2}}$. Analogous to [13, Chapter II, §3] we put $P:=A_{1 / 2}(T e)-2\left[A_{1 / 2}(T e)\right]^{2}$ and get $\left[P, A_{1 / 2}(T e)\right]=$ 0 , Trace $P^{2}=0, P^{\sigma}=P$. Hence $P=0$. It follows that $\left[A_{1 / 2}(T e), \hat{T}\right]=0$. Further $A_{1 / 2}(T e)-\hat{T}$ is skew symmetric with respect to $\sigma$. The last two assertions together with the fact that $\hat{T}$ has only real eigenvalues, force $A_{1 / 2}(T e)=\hat{T}$. Now it is easy to check that only (e) remains to be proved. 
(3) For (e) we may assume $2 \leqslant i<j \leqslant r$ by [5a, Satz 5.2]. Therefore we have $T\left(x_{i j}\right) \mathfrak{A}_{k} \subset \mathfrak{A}_{k}, k=0, \frac{1}{2}, 1$ and $T\left(x_{i j}\right) \mathfrak{A}_{1}=0$. So we may view $T\left(x_{i j}\right)$ as a pair $\left(T_{0}\left(x_{i j}\right), \hat{T}\right) \in$ End $\mathfrak{U}_{0} \times$ End $\mathfrak{A}_{1 / 2}$, where $T_{0}\left(x_{i j}\right)=\left.2 A_{d_{i}}\left(x_{i j}\right)\right|_{\mathfrak{x}_{0}}$. Now the relation (c) of Lemma 3.6 for $\left(T_{0}\left(x_{i j}\right), \hat{T}\right)$ reads $A_{1 / 2}\left(\left[T_{0}\left(x_{i j}\right)\right]^{o} d_{i}\right)=\hat{T}^{o} A_{1 / 2}\left(d_{i}\right)$ $+A_{1 / 2}\left(d_{i}\right) \hat{T}$. But $\left[T_{0}\left(x_{i j}\right)\right]^{\sigma} d_{i}=0$, so $2 A_{1 / 2}\left(x_{i j}\right) A_{1 / 2}\left(d_{i}\right)=\left[\hat{T}, A_{1 / 2}\left(d_{i}\right)\right]$. Because of $a \mapsto V, T \mapsto T e$ being an isomorphism we get $\left[T\left(x_{i j}\right), A\left(d_{i}\right)\right]=$ $\left[T\left(x_{i j}\right), T\left(d_{i}\right)\right]=T\left(T_{0}\left(x_{i j}\right) d_{i}-T\left(d_{i}\right) x_{i j}\right)=T\left(x_{i j}-\frac{1}{2} x_{i j}\right)=\frac{1}{2} T\left(x_{i j}\right)$. So we conclude $4 A_{1 / 2}\left(x_{i j}\right) A_{1 / 2}\left(d_{i}\right)=\hat{T}$. Finally we use the definition of $A_{d_{i}}\left(x_{i j}\right)$ and Lemma 3.6 for $T=A\left(d_{i}\right)$ to get $\left.2 A_{d_{i}}\left(x_{i j}\right)\right|_{\mathscr{A}_{1 / 2}}=4 A_{1 / 2}\left(x_{i j}\right) A_{1 / 2}\left(d_{i}\right)=\hat{T}$.

To prove the following corollary we use Theorem 5.1 and results of [13, Chapter II]. For notation concerning left-symmetric algebras see [5a].

COROllary 5.2. Let a be a ctt-algebra for $F$. Denote by $\mathcal{L}$ the left-symmetric algebra associated to a. Then

(a) $T \in$ a, $T^{2} e=T e$ implies $T=A(T e)$.

(b) The Peirce-decomposition of Theorem 5.1 is a "normal decomposition" of the left-symmetric algebra $\mathcal{L}_{\sigma}$.

Proof. Part (b) is easily checked by using Theorem 5.1 and the fact that $T(u)$ is the left multiplication in the left symmetric algebra $\mathcal{E}$ associated to $a$. To prove (a), we note that $T^{2} e=T e$ implies that $q:=T e$ is an idempotent of . But then [13, Chapter II, Proposition 9] shows that $q$ is a sum of some $d_{i}$ 's. The assertion now follows from Theorem 5.1.

The next two corollaries explain some consequences of Theorem 5.1 for $\mathfrak{X}_{F}$ (for definition see $\$ 1.2$ ).

To point out that the following results are true for all triples $F$ of $\mathscr{F}$ we use again the triple designator as index.

COROLlaRy 5.3. For all triples $F$ of $\mathscr{F}$ there exists a CSI $d_{1}, \ldots, d_{r}$ of $\mathfrak{X}_{F}(r$ depending on $F$ ) such that $d_{i} \in \mathfrak{X}_{F}$ and $\mathfrak{X}_{1}\left(d_{i}\right)=\mathbf{R} d_{i}$.

The next result has partially been proved in [14].

COROLlary 5.4. Let $F$ be a triple of $\mathcal{F}_{\text {then }} \mathfrak{X}_{F}$ is a formally-real Jordan-algebra and $Y_{F}:=\left\{x \in K_{F} ; H_{F}(x) \in \operatorname{Aut}\left(K_{F}, \eta_{F}\right)\right\}$ its positive cone. Further $V_{F}$ is a Jordan-bimodule with respect to $\mathfrak{X}_{F}$.

Proof. To prove that $\mathfrak{X}$ is a subalgebra we essentially proceed as in [14, Chapter IV, §3]. Let $\Gamma$ be a ctt-subgroup of $\operatorname{Aut}(K, \eta)$; then we form a Peirce-decomposition associated to Lie $\Gamma$ according to Theorem 5.1. For $x \in \mathfrak{X}$ we get $\left[A\left(d_{i}\right), A(x)\right] \in \operatorname{Lie} \operatorname{Aut}(K, \eta) \cap \operatorname{Der} \mathfrak{A}$ for $\left[A\left(d_{i}\right), A(x)\right] e=0$ implies $\left[A\left(d_{i}\right), A(x)\right]$ to be an element of Der $\mathfrak{A}$ by [5a, (1.18)]. Hence $\left[A\left(d_{i}\right)\right.$, $A(x)] \mathfrak{X} \subset \mathfrak{X}$. From this we conclude $\mathfrak{X}=\bigoplus\left(\mathfrak{X} \cap \mathfrak{U}_{i j}\right)$. Using $\left[A\left(d_{i}\right), A\left(x_{i j}\right)\right] x_{j k}$ $\in \mathfrak{X}$ for all $x_{i j}, x_{j k} \in \mathfrak{X}$, we get $x_{i j} x_{j k} \in \mathfrak{X}$. Hence $\mathfrak{X}$ is a subalgebra and it is 
Jordan because of [5a, Satz 2.4]. Consider now the map $\mathfrak{X} \rightarrow Y, x \mapsto$ $\exp A(x) e$. It is a bijection by [5a, Lemma 2.1]. Further, $Y$ is the positive cone of $\mathfrak{X}$ by [1, XI, Satz 3.7]. Finally, compare [5a, Satz 3.1] with the definition of a Jordan-bimodule in [6].

To finish this series of corollaries we compare with [2].

COROLlaRy 5.5. Let $F$ be a triple of $\mathcal{F}$ and a a ctt-algebra for $F$. Then the associated Peirce-decomposition of Theorem 5.1 is a " $r$ - $\AA$-Zerlegung" $\mathcal{C}$ of $\mathfrak{A}$. Further $\mathfrak{a}=\mathfrak{g}^{\mathcal{e}}$ and $K_{F}=Y_{\mathcal{E}}$

6. Splitting of ctt-algebras. We keep in mind the definitions of $\$ 5.1$.

1. In this subsection we fix a triple $F=\langle K, \eta, e\rangle$ of $\mathscr{F}$ and an idempotent $p$ of $\subseteq=\Im_{F}$. We form $F_{i}$ and $\delta_{i}$ with respect to $p$ according to (2.3) and §3.1.

First we describe the splitting of a ctt-algebra for a triple $F$ of $\mathscr{F}$.

THeOREM 6.1. Let a be a subspace of Lie $\operatorname{Aut}(K, \eta)$. Then a is a ctt-algebra for $F$ that is contained in $b$ if and only if there exist Lie-subalgebras $a_{i} \subset \delta_{i}$, $i=0,1$, such that

(a) $a_{i}$ is trigonalizable.

(b) $a=a_{1}+b_{1 / 2}+a_{0}$ (direct sum of vectorspaces),

(c) $\mathfrak{a}_{i i}:=\left\{\left.T\right|_{\mathfrak{A}_{i}} ; T \in \mathfrak{a}_{i}\right\}, i=0,1$, is a ctt-algebra for $F_{i}$.

Proof. (1) Let $a \subset \delta$ be a ctt-algebra for $F$. Then by Lemma 3.1 every $T \in a$ can be written $T=T_{1}+T_{1 / 2}+T_{0}, T_{i} \in \delta_{i}$. Let $a^{\prime}:=\left\{T \in a ; T_{1 / 2}\right.$ $=0\}=\left\{T \in \mathfrak{a} ; T e \in \mathfrak{A}_{1}+\mathfrak{A}_{0}\right\}, \mathfrak{A}_{i}=\mathfrak{A}_{i}(p)$. Then it is easy to see that $\mathfrak{a}_{i i}^{\prime}:=\left\{T \mid \mathfrak{A}_{i} ; T \in \mathfrak{a}^{\prime}\right\}$ is a trigonalizable subalgebra of Lie $\operatorname{Aut}\left(K_{i}, \eta_{i}\right), i=0$, 1 .

(2) $\mathfrak{a}_{i i}^{\prime}$ is a ctt-algebra for $F_{i}, i=0,1$, for the mapping $T \mapsto T y$ from a to $V$ is for all $y \in K_{1}+K_{0}$ surjective. Especially we have

(3) Let $T \in \mathfrak{a}, T e \in \mathfrak{U}_{i}, i=0,1$. Then $T \in \mathfrak{a}^{\prime}$; further, $T_{1} p_{1}=0$ if $i=0$ and $T_{0} p_{0}=0$ if $i=1$. From (2) we conclude $T_{11}=\left.T_{1}\right|_{a_{1}}=0$ if $i=0$ and $T_{00}=\left.T_{0}\right|_{\mathfrak{A}_{0}}=0$ if $i=1$. We are now going to show

(4) Let $T \in \mathfrak{A}, T e \in \mathfrak{A}_{i}, i=0,1$. Then $T \in \mathfrak{d}_{i}$. The case $i=0$ is easy, for, from (3), we have $T_{11}=0$ and so $T_{1}=0$ by Lemma 3.1. Now let $i=1$. First we apply Theorem 5.1 to $a_{11}^{\prime}$ and get a description of the elements of $a_{11}^{\prime}$ in terms of a Peirce-decomposition $\mathfrak{A}_{1}=\bigoplus_{1<i<j<s} \mathfrak{B}_{i j}$ of $\mathfrak{A}_{1}$. Now, for the elements $T \in a^{\prime}$ that satisfy $T p_{0}=0$ we may assume without loss of generality $T p_{1} \in \mathfrak{B}_{i j}$ and can take over-in essentially only interchanging 0 and 1-parts (2) and (3) of the proof of Theorem 5.1. So we see that $T$ itself, not only $\left.T\right|_{\mathfrak{A}_{1}}$, is of the form $A_{d_{i}}\left(x_{i j}\right), x_{i j} \in \mathfrak{B}_{i j}, d_{i} \in \mathfrak{B}_{i i}$. It follows $T \in \delta_{1}$ and (4) is proved.

(5) We define $a_{i}:=\left\{T \in a ; T e \in \mathscr{A}_{i}\right\}$ and show $a^{\prime}=a_{1}+a_{0}$. For $T \in a^{\prime}$ we have $T e=x_{1}+x_{0} \in \mathfrak{U}_{1}+\mathfrak{U}_{0}$. Hence by (4) there exist $T_{i} \in \delta_{i} \cap$ a such 
that $T_{i} e=x_{i}$. So $\left(T-T_{1}-T_{0}\right) e=0$ and $T=T_{1}+T_{0}$ follows from $\S 5.1$.

Thus we have shown $a^{\prime} \subset a_{1}+a_{0}$. The other inclusion is trivial.

Now, it is easy to check that only $\delta_{1 / 2} \subset$ a remains to be proved.

(6) As ctt-algebras are maximal trigonalizable we have Id $\in a_{11}$; hence by Lemma 3.1 we conclude $A(p) \in a_{1}$. Now, let $T \in \mathfrak{a}, T e=x_{1 / 2} \in \mathfrak{X}_{1 / 2}$. We write $T=T_{1}+T_{1 / 2}+T_{0}$ with $T_{i} \in \mathrm{\delta}_{i}$ and get a $\ni[T, A(p)]=\left[T_{1 / 2}, A(p)\right]$ $=A_{p}\left(T_{1 / 2} p\right)=A_{p}\left(x_{1 / 2}\right)=\frac{1}{2} T_{1 / 2}$; by (3.2) and [10, IV, Corollary 8]. So $T-$ $T_{1 / 2} \in a$ and $\left(T-T_{1 / 2}\right) e=0$. This forces, as above, $T=T_{1 / 2}$.

(7) Let us now assume that a satisfies (a), (b), and (c) with $a_{i} \subset \delta_{i}$ as in the theorem. Then by (b) and Lemma 3.1 it is easy to see that $a$ is a Lie-algebra. Further, $a^{\prime}:=a_{1}+a_{0}$ is a subalgebra of $a$. As all $T_{i} \in a_{i}$ have only real eigenvalues, then, because of $\left[a_{1}, a_{0}\right]=0$, all $T_{1}+T_{0} \in a^{\prime}$ have only real eigenvalues. Hence $a^{\prime}$ is trigonalizable by [12]. From this we conclude that $\mathfrak{b}_{i}:=\left.\mathfrak{a}^{\prime}\right|_{\mathfrak{A}_{i}}, i=0, \frac{1}{2}, 1$, is trigonalizable. Patching together the trigonalizing bases of $\mathfrak{b}_{0}, \mathfrak{b}_{1 / 2}, \mathfrak{b}_{1}$ we get a trigonalizing basis for $a$. To see the ctt-property use (c) and Theorem 1.8.

For the next corollary we recall that $p$ is an idempotent of $\subseteq=\Im_{F}$ and $c$ the unit of $\subseteq$. We put $c_{1}=c$ and $c_{0}=e-c$.

COROLlARY 6.2. Let a be a ctt-algebra for $F$.

(a) If a $\subset \delta^{p}$, then $A(p) \in \mathfrak{a}$.

(b) $A(c) \in$ a.

Proof. Part (a) is clear from part (6) of the proof of Theorem 6.1.

Part (b) follows from part (a) because of $\delta^{c}=\operatorname{Lie} \operatorname{Aut}(K, \eta)$.

Corollary 6.3. In Theorem 5.1 it is possible to reorder the CSI $d_{1}, \ldots, d_{r}$ such that the first $s$ idempotents ( $s \leqslant r$ properly choosen) form a CSI of $\subseteq$.

Proof. Under the hypothesis of Theorem 5.1, we get, by Theorem 6.1, for $p=c$ algebras $\mathfrak{a}_{i i}$ that again satisfy the hypothesis of Theorem 5.1. Applying Theorem 5.1 to $\mathfrak{a}_{i i}$, we get a CSI $d_{1}, \ldots, d_{s}$ of $\mathfrak{S}=\mathfrak{A}_{1}=\mathfrak{A}_{1}(c)$ and a CSI $d_{s+1}, \ldots, d_{r}$ of $\mathfrak{A}_{0}$. We now proceed nearly word for word as in parts (2) and (3) of the proof of Theorem 5.1 verifying the assertions of Theorem 5.1 for $d_{1}, \ldots, d_{r}$. Finally by $\mathfrak{A}_{i i}=\mathbf{R} d_{i}$ and the Corollary of [13, II, Proposition 9], the assertion of Corollary 6.3 follows.

2. In this section we derive a general splitting property for a triple $F$ of $\mathscr{F}$. So let $F$ be a triple of $\mathscr{F}$ and a a ctt-algebra for $F$. Futher let $T \in$ a such that $T^{2} e=T e$. Then by Corollary 5.2 we know $d:=T e$ to be an idempotent of $\mathfrak{X}$. Hence we may form the Peirce-decomposition $\mathfrak{U}=\mathfrak{A}_{1}+\mathfrak{A}_{1 / 2}+\mathfrak{X}_{0}$ of $\mathfrak{A}$ with respect to $d$. Denote by $\pi_{i}$ the canonical projection of $\mathfrak{A}$ onto $\mathfrak{A}_{i}$. Analogous to $\$ 2$ we put $K_{i}:=\pi_{i}(K), i=0,1$, and define $a_{1}:=\left\{T \in \mathfrak{a} ;\left.T\right|_{\mathfrak{x}_{0}}=0\right.$, $\left.T \mathfrak{A}_{i} \subset \mathfrak{A}_{i}, i=1, \frac{1}{2}\right\}$ and $\mathrm{a}_{0}:=\left\{T \in \mathfrak{a} ;\left.T\right|_{\mathfrak{A}_{1}}=0, T \mathfrak{A}_{i} \subset \mathfrak{A}_{i}, i=0, \frac{1}{2}\right\}$. Using this notation we get 
LEMMA 6.4. (a) $K_{i}$ is a regular cone in $\mathfrak{A}_{i}$.

(b) The group generated by $a_{i i}:=\left\{\left.T\right|_{\mathscr{A}_{i}} ; T \in a_{i}\right\}$ operates transitively on $K_{i}$.

(c) $K_{1}+K_{0} \subset K$.

Proof. (a) Because of $A(d) \in \operatorname{Lie} \operatorname{Aut}(K, \eta)$ we may proceed as in [10, IV, Theorem 17].

(c) By hypothesis, a is a ctt-algebra for $F$, hence there exist idempotents $d_{1}, \ldots, d_{r} \in \mathfrak{X}$ such that the assertions of Theorem 5.1 hold. Because of $d=T e=T^{2} e$, we find, by Corollary 5.2 and [13, II, Proposition 9], a subset $I \subset\{1, \ldots, r\}$ such that $d=\Sigma_{i \in I} d_{i}$. From this we get $e-d=\Sigma_{i \in J} d_{i}$, $J=\{1, \ldots, r\} \backslash I$. And by Corollary 6.3 we split $d=f_{1}+f_{3}, e-d=f_{2}+$ $f_{4}$ with $f_{1}, f_{2} \in \mathfrak{S}=\mathfrak{A}_{1}(c)$ such that $f_{1}+f_{2}=c_{1}$. Then $f_{3}, f_{4} \in \mathfrak{U}_{0}(c) \cap \mathfrak{X}$, $f_{3}+f_{4}=e-c=c_{0}$. Using [5a] we may form the Peirce-decomposition $\mathfrak{U}=\bigoplus_{1<i<j<4^{\mathfrak{A}}}$ of $\mathfrak{A}$ with respect to $f_{1}, \ldots, f_{4}$. We easily get

$$
\begin{aligned}
\mathfrak{U}_{1}(c) & =\mathfrak{U}_{11}+\mathfrak{U}_{12}+\mathfrak{A}_{22}, \\
\mathfrak{A}_{1 / 2}(c) & =\mathfrak{U}_{13}+\mathfrak{U}_{14}+\mathfrak{A}_{23}+\mathfrak{A}_{24}, \\
\mathfrak{U}_{0}(c) & =\mathfrak{U}_{33}+\mathfrak{A}_{34}+\mathfrak{U}_{44} .
\end{aligned}
$$

Now, let $x \in K$, then by Theorem 1.8

$$
\begin{aligned}
& x=x_{1}+x_{1} x_{1 / 2}+x_{0}+\frac{1}{2} c_{0}\left(x_{1 / 2} \cdot x_{1} x_{1 / 2}\right), \\
& \quad \text { for } x_{1} \in K_{1}^{c_{1}}, x_{0} \in K_{1}^{c_{0}}, x_{i} \in \mathfrak{A}_{i}(c) .
\end{aligned}
$$

Here we insert (1); then for the projection $\pi_{1}(x)$ a straightforward computation shows

$$
\begin{aligned}
\pi_{1}(x)= & x_{11}+x_{11} x_{13}+x_{12} x_{23}+x_{33} \\
& +\frac{1}{2} c_{0}\left(x_{11} x_{13} \cdot x_{13}+x_{12} x_{13} \cdot x_{23}+x_{12} x_{23} \cdot x_{13}+x_{22} x_{23} \cdot x_{23}\right) .
\end{aligned}
$$

Analogously we get for $\pi_{0}(y), y \in K$,

$$
\begin{aligned}
\pi_{0}(y)= & y_{22}+y_{12} y_{14}+y_{22} y_{24}+y_{44} \\
& +\frac{1}{2} c_{0}\left(y_{11} y_{14} \cdot y_{14}+y_{12} y_{14} \cdot y_{24}+y_{12} y_{24} \cdot y_{14}+y_{22} y_{24} \cdot y_{24}\right) .
\end{aligned}
$$

What we intend to show is $\pi_{1}(x)+\pi_{0}(y) \in K$ for all $x, y \in K$. Let us abbreviate $\pi_{1}(x)+\pi_{0}(y):=z_{1}+z_{1 / 2}+z_{0}, z_{i} \in \mathfrak{U}_{i}\left(c_{1}\right)$. Then by Theorem 1.8 we have $z \in K$ if and only if

$$
z_{1} \in K_{1}^{c_{1}} \text { and } z_{0}-\frac{1}{2} c_{0}\left(z_{1 / 2} \cdot\left[A_{1 / 2}\left(z_{1}\right)\right]^{-1} z_{1 / 2}\right) \in K_{0}^{c_{1}}
$$

It is clear that the first condition is satisfied because of Corollary 1.9 applied to $K_{1}^{c_{1}}$. To verify the second condition we use the composition rules for a Peirce-decomposition, $[2, \S 1]$, and the identity $c_{0}\left(x_{1 / 2} \cdot x_{1} y_{1 / 2}\right)=$ $c_{0}\left(x_{1} x_{1 / 2} \cdot y_{1 / 2}\right)$ for all $x_{1 / 2}, y_{1 / 2} \in \mathfrak{U}_{1 / 2}(c), x_{1} \in \mathfrak{U}(c),[2, \S 2]$, to get 


$$
\begin{aligned}
& z_{0}-\frac{1}{2} c_{0}\left(z_{1 / 2} \cdot\left[A_{1 / 2}\left(z_{1}\right)\right]^{-1} z_{1 / 2}\right)=x_{33}+y_{44} \\
& +\frac{1}{2} c_{0}\left(x_{22} x_{23} \cdot x_{23}-\left(x_{12} x_{23}\right) \cdot\left[A_{1 / 2}\left(x_{11}\right)\right]^{-1}\left(x_{12} x_{23}\right)\right. \\
& \left.\quad+y_{11} y_{14} \cdot y_{14}-\left(y_{12} y_{14}\right) \cdot\left[A\left(y_{22}\right)\right]^{-1}\left(y_{12} y_{14}\right)\right) .
\end{aligned}
$$

Now, by (1) and (2) we have $x_{1}=x_{11}+x_{12}+x_{22} \in K_{1}^{c_{1}}$ and by Theorem 1.8 , applied to $K_{1}^{c_{1}}$ and $p:=f_{1}$, we may replace this expression for $x_{1}$ by $x_{1}=x_{11}+x_{11} q_{12}+q_{22}+\frac{1}{2} f_{2}\left(q_{12} \cdot x_{11} q_{12}\right)$. From this we conclude, using [2, Satz 2.1],

$$
\begin{aligned}
c_{0}([ & \left.\left(q_{22}+\frac{1}{2} f_{2}\left(q_{12} \cdot x_{11} q_{12}\right)\right) x_{23}\right] \\
\quad & \left.\quad-\left[\left(x_{11} q_{12}\right) x_{23}\right] \cdot\left[A_{1 / 2}\left(x_{11}\right)\right]^{-1}\left[\left(x_{11} q_{12}\right) x_{23}\right]\right) \\
\quad= & c_{0}\left(q_{22} x_{23} \cdot x_{23}\right)+c_{0}\left(\left[\left(x_{11} q_{12}\right) x_{23}\right] \cdot\left[q_{12} x_{23}\right]-\left[\left(x_{11} q_{12}\right) x_{23}\right] \cdot\left(q_{12} x_{23}\right)\right) \\
\quad= & c_{0}\left(q_{22} x_{23} \cdot x_{23}\right) .
\end{aligned}
$$

Because of $q_{22}$ being a square in $\mathfrak{A}_{1}(c)$ we see that this term is in $\bar{K}_{0}$. Analogously we use Theorem 1.8 for $K_{1}^{c_{1}}$ and $p:=f_{2}$ to see that the similar sum of terms of (6) that are built up of $y_{i j}$ 's lies in $\bar{K}_{0}$. Now the assertion (c) is easily proved by induction on $n=\operatorname{dim} \mathfrak{A}$.

(b) We form the Peirce-decomposition of $\mathfrak{A}$ with respect to $d_{1}, \ldots, d_{r}$. Then $T\left(x_{i j}\right) \in a_{1}$ if and only if $i \leqslant j, i, j \in I$ and $T\left(x_{i j}\right) \in a_{0}$ if and only if $i<j, i, j \in J$. Further $a=a_{1}+a_{1 / 2}+a_{0}$ where $a_{1 / 2}:=\{T \in a ; T e \in$ $\left.\mathfrak{U}_{1 / 2}\right\}$. Now, $a \rightarrow V, T \mapsto T x$ is surjective for all $x \in K_{1}+K_{0}$, hence $a_{i i} \rightarrow a_{i}$, $T \mapsto T x_{i}$ is surjective for all $x_{i} \in K_{i}$. This proves (b).

Because of part (c) of Lemma 6.4 we may define $\eta_{1}: K_{1} \rightarrow \mathbf{R}^{+}, x_{1} \mapsto \eta\left(x_{1}\right.$ $+e-d)$ and $\eta_{0}: K_{0} \rightarrow \mathbf{R}^{+}, x_{0} \mapsto \eta\left(d+x_{0}\right)$. $\mathscr{F}$.

Corollary 6.5. $F_{1}:=\left\langle K_{1}, \eta_{1}, d\right\rangle$ and $F_{0}:=\left\langle K_{0}, \eta_{0}, e-d\right\rangle$ are triples of

Proof. We have to check (1.1) to (1.5). But (1.1) is obvious and (1.2) is clear from $A(d) \in \operatorname{Lie} \operatorname{Aut}(K, \eta)$. (1.3) is straightforward and (1.5) is clear from Lemma 6.4(b). Let $x_{n} \in K_{1}, x_{n} \rightarrow x \in$ boundary of $K_{1}$; then $x+e-d$ $\in$ boundary of $K$ in view of the definition of $K_{1}$ and $\eta_{1}\left(x_{n}\right)$ converges to $+\infty$. The rest is proved in a similar manner.

7. Injectivity of $A_{1 / 2}$. Let $F$ be a triple of $\mathfrak{F}$ and $p \in \mathfrak{S}_{F}, p^{2}=p$. Then there are two mappings $A_{1 / 2}$ defined by (1.15).

1. Look at $2 A_{1 / 2}: \mathfrak{A}_{1}(p) \rightarrow$ End $\mathfrak{A}_{1 / 2}(p)$; this is, by [2] and Theorem 1.2, a homomorphism of Jordan-algebras. Hence its kernel is an ideal of $\mathfrak{A}_{1}(p)$. Obviously $\operatorname{Ker} A_{1 / 2}$ is an ideal of $\mathfrak{A}$ and a formally-real Jordan-algebra. So, by Theorem 4.1, the triple $F$ is reducible and we have proved 
THEOREM 7.1. If the triple $F$ is not reducible then $A_{1 / 2}: \mathfrak{A}_{1}(p) \rightarrow$ End $\mathfrak{A}_{1 / 2}(p)$ is injective.

2. Let us now consider $A_{1 / 2}: \mathfrak{A}_{0}(p) \rightarrow$ End $\mathfrak{A}_{1 / 2}(p)$. As usual we write $\mathfrak{A}_{i}$ instead of $\mathfrak{A}_{i}(p)$ and form $M:=\left\{p_{0} \cdot x_{1 / 2}^{2} ; x_{1 / 2} \in \mathfrak{A}_{1 / 2}\right\}, p_{0}:=e-p$. Further we denote by $\langle M\rangle$ the vectorspace generated by $M$. Then using Lemma 3.6 we easily get

LEMMA 7.2. (a) $\operatorname{Ker} A_{1 / 2}=M^{\perp}=\left\{x_{0} \in \mathfrak{A}_{0} ; \sigma\left(x_{0}, m\right)=0\right.$ for all $\left.m \in M\right\}$. (b) $T^{\sigma} \operatorname{Ker} A_{1 / 2} \subset \operatorname{Ker} A_{1 / 2}, T\langle M\rangle \subset\langle M\rangle$ for all $T \in \delta_{0}$.

Since $\mathfrak{A}_{0}=\operatorname{Ker} A_{1 / 2} \oplus\langle M\rangle$, we may decompose $p_{0}=d_{1}+d_{2}, d_{1} \in$ $\operatorname{Ker} A_{1 / 2}, d_{2} \in\langle M\rangle$. For the following we fix a trigonalizable subalgebra $a_{0}$ of $\delta_{0}$ such that $a_{00}:=\left\{\left.T\right|_{\mathfrak{A}_{0}} ; T \in a_{0}\right\}$ is a ctt-algebra for $F_{0}$. As before, by $T\left(x_{0}\right), x_{0} \in \mathfrak{A}_{0}$, we denote the unique element $T$ of $a_{0}$ such that $T e=x_{0}$. We have $T\left(T\left(x_{0}\right) y_{0}-T\left(y_{0}\right) x_{0}\right)=\left[T\left(x_{0}\right), T\left(y_{0}\right)\right]$ for all $x_{0}, y_{0} \in \mathfrak{A}_{0}$.

LEMMA 7.3. (a) $T\left(x_{0}\right) z_{0}=0$ for all $x_{0} \in \operatorname{Ker} A_{1 / 2}, z_{0} \in\langle M\rangle$.

(b) $A\left(d_{i}\right) \in a_{0}, i=0,1$.

Proof. As usual we abbreviate $T_{1 / 2}\left(x_{0}\right):=\left.T\left(x_{0}\right)\right|_{\mathfrak{A}_{1 / 2}}$. Then we have $T_{1 / 2}\left(x_{0}\right)=A_{1 / 2}\left(x_{0}\right)+Q_{1 / 2}, Q_{1 / 2}\left(x_{0}\right)^{\sigma}=-Q_{1 / 2}\left(x_{0}\right)$ because of $T+T^{\sigma}=$ $2 A(T e)$ for $T \in \mathcal{D}$. Hence $T_{1 / 2}\left(x_{0}\right)$ is skew adjoint for $x_{0} \in \operatorname{Ker} A_{1 / 2}$. As $\left\{T_{1 / 2} ; T \in a_{0}\right\}$ is trigonalizable we conclude $T_{1 / 2}\left(x_{0}\right)=0$ for $x_{0} \in \operatorname{Ker} A_{1 / 2}$. Now, observe Lemma 3.6 and [2, Lemma 2.4], to see $T\left(x_{0}\right)\left(p_{0} \cdot x_{1 / 2}^{2}\right)=$ $2 p_{0}\left(x_{1 / 2} \cdot T_{1 / 2}\left(x_{0}\right) x_{1 / 2}\right)=0$. Further, $T\left(d_{2}\right) d_{2}=T\left(d_{1}+d_{2}\right) d_{2}=d_{2}$. Therefore $T\left(d_{1}\right) d_{1}=d_{1}$ and the lemma is proved because of Corollary 5.2.

By Lemma 7.3 we get $d_{i} \in \mathfrak{X}=\mathfrak{X}_{F}$. Hence we may form the Peirce-decomposition $\mathfrak{A}_{0}=\tilde{\mathfrak{A}}_{0}+\tilde{\mathfrak{A}}_{1 / 2}+\tilde{\mathfrak{A}}_{0}, \tilde{\mathfrak{A}}_{i}=\left(\mathfrak{A}_{0}\right)_{i}\left(d_{1}\right)$, of $\mathfrak{A}_{0}$ with respect to $d_{1}$. We are now going to prove

LEMMA 7.4. (a) Ker $A_{1 / 2}=\tilde{\mathfrak{X}}_{1}+\tilde{\mathfrak{X}}_{1 / 2},\langle M\rangle=\tilde{\mathfrak{A}}_{0}$.

(b) $T\left(\tilde{\mathfrak{A}}_{i}\right) \tilde{\mathfrak{A}}_{j} \subset \tilde{\mathfrak{A}}_{j}$ for $i=0,1$ and $j=0, \frac{1}{2}, 1$.

(c) $T\left(\tilde{\mathfrak{A}}_{1 / 2}\right) \tilde{\mathfrak{A}}_{1} \subset \tilde{\mathfrak{A}}_{1 / 2}, T\left(\tilde{\mathfrak{A}}_{1 / 2}\right) \tilde{\mathfrak{A}}_{1 / 2} \subset \tilde{\mathfrak{A}}_{0}, T\left(\tilde{\mathfrak{A}}_{1 / 2}\right) \tilde{\mathfrak{A}}_{0}=0$.

Proof. (1) We have $\langle M\rangle \subset \tilde{\mathfrak{A}}_{0}$ because of $d_{1}\left(p_{0} \cdot x_{1 / 2}^{2}\right)=T\left(d_{1}\right)\left(p_{0} \cdot x_{1 / 2}^{2}\right)$ $=2 p_{0}\left(x_{1 / 2} \cdot T_{1 / 2}\left(d_{1}\right) x_{1 / 2}\right)=0$.

(2) Now let $S:=\left\{x \in \operatorname{Ker} A_{1 / 2} ; d_{1} x=0\right\}$; then $T(x) x=0$ for all $x \in S$. For $T\left(d_{2}\right) T(x)=T\left(T\left(d_{2}\right) x-T(x) d_{2}\right)+T(x) T\left(d_{2}\right)$ implies $d_{2}(T(x) x)=T(x$ $-0) x+T(x) x=2 T(x) x$ by Lemma 7.3 and $d_{2} x=\left(p_{0}-d_{1}\right) x=x$. Because $A\left(d_{2}\right)$ has at most the eigenvalues $0, \frac{1}{2}, 1$ the assertion follows (see [5a, §3]).

(3) We show $S=0$. First observe $\operatorname{Ker} A_{1 / 2} \ni T(x)^{\circ} x=2 A(x) x-T(x) x$ $=2 x^{2}$ to conclude that $S$ is a subalgebra of $\tilde{\mathfrak{A}}_{0}$. As $S \rightarrow \mathbf{R}, s \mapsto \sigma(e, s)$ is an 
associative linear form on $S$ we see that $S$ has a unit $q$. But now $0=\sigma\left(d_{2}\right.$, $q)=\sigma\left(d_{2}, q^{2}\right)=\sigma\left(d_{2} q, q\right)=\sigma(q, q)$ and $S=0$ follows. Thus (a) is proved.

To show (b) we first note that from (a) and Lemma 7.2, 7.3 we get

(4) $T\left(\tilde{\mathfrak{A}}_{0}\right) \tilde{\mathfrak{A}}_{0} \subset \tilde{\mathfrak{A}}_{0}, T\left(\tilde{\mathfrak{A}}_{1 / 2}+\tilde{\mathfrak{A}}_{1}\right) \tilde{\mathfrak{A}}_{0}=0$.

Choose $x \in \tilde{\mathfrak{A}}_{i}$ and $y \in \tilde{\mathfrak{A}}_{j}$. Then

$$
\begin{aligned}
(1-j) T(x) y & =T(x) T\left(d_{2}\right) y=T\left(d_{2}\right) T(x) y+T\left(T(x) d_{2}-d_{2} x\right) y \\
& =d_{2} \cdot T(x) y+T\left(T(x) d_{2}\right) y-(1-i) T(x) y .
\end{aligned}
$$

From this we conclude

(5) $(2-i-j) T(x) y=d_{2} \cdot T(x) y+T\left(T(x) d_{2}\right) y$.

For $i=\frac{1}{2}, 1$ we have $x \in \operatorname{Ker} A_{1 / 2}$. Hence the last term in (5) vanishes and by exploiting (5) it is easy to check (c) and (b) for $i=1$. So we just have to demonstrate $T\left(\tilde{\mathfrak{A}}_{0}\right) \tilde{\mathfrak{A}}_{j} \subset \tilde{\mathfrak{A}}_{j}$. Let $x \in \tilde{\mathfrak{A}}_{0}$. Then $T(x) d_{1}=-T(x)^{\circ} d_{1} \in$ $\operatorname{Ker} A_{1 / 2}$; but $T(x) d_{1}=x-T(x) d_{2} \in \tilde{\mathfrak{A}}_{0}=\langle M\rangle$ by (4). So $T(x) d_{1}=0$ and $x=T(x) d_{2}$ for all $x \in \tilde{\mathfrak{A}}_{0}$. Inserting this in (5) we prove the lemma.

THEOREM 7.5. The following conditions are equivalent:

(a) $\delta^{p}=\operatorname{Lie} \operatorname{Aut}(K, \eta)$ and $A_{1 / 2}: \mathfrak{U}_{0}(p) \rightarrow$ End $\mathfrak{U}_{1 / 2}(p)$ is injective,

(b) $\mathfrak{A}_{1}(p)=\subseteq$.

Proof. The part (a) $\Rightarrow(b)$ is easily seen from Theorem 3.3. To prove the converse we use Lemma 7.4 and choose a basis in $\tilde{\mathfrak{A}}_{j}, j=0, \frac{1}{2}, 1$, that trigonalizes $\left.T\left(\tilde{\mathfrak{A}}_{1}+\tilde{\mathfrak{A}}_{0}\right)\right|_{\mathfrak{A}_{0}}$. Patching these bases of $\tilde{\mathfrak{A}}_{0}, \tilde{\mathfrak{A}}_{1 / 2}, \tilde{\mathfrak{A}}_{1}$, we get a trigonalizing basis of $a_{00}$. If $\operatorname{Ker} A_{1 / 2} \neq 0$, then $\tilde{\mathfrak{A}}_{1} \neq 0$ and by Korollar 1 of [5a, Satz 5.2] there exists a $0 \neq d \in \widetilde{S}_{F_{0}} \cap \tilde{\mathfrak{A}}_{1}$ satisfying $d^{2}=d$ and $\left.A_{d}\left(x_{0}\right)\right|_{\mathfrak{x}_{0}}$ $\in \mathfrak{a}_{00}$ for all $x_{0} \in \mathfrak{A}_{0}$. We are going to prove $d \in \mathfrak{S}$, a contradiction to (b).

(1) First we show $A_{d}(x)=0$ for all $x \in \mathfrak{A}_{1}+\mathfrak{A}_{1 / 2}$. Here the case $x \in \mathfrak{A}_{1}$ is trivial. For $x=x_{1 / 2} \in \mathfrak{U}_{1 / 2}$ we have $A_{d}\left(x_{1 / 2}\right)=\left[A\left(x_{1 / 2}\right), A(d)\right]$ because of $d \in \operatorname{Ker} A_{1 / 2}$. So $A_{d}\left(x_{1 / 2}\right) y=0$ for $y \in \mathfrak{U}_{1}+\mathfrak{U}_{1 / 2}$ by $\langle M\rangle=\tilde{\mathfrak{A}}_{0}, d \in \tilde{\mathfrak{A}}_{1}$. Now, let $y=\tilde{y}_{1}+\tilde{y}_{1 / 2}+\tilde{y}_{0} \in \mathfrak{A}_{0}$. Then $A_{d}\left(x_{1 / 2}\right) y=x_{1 / 2}\left(d \tilde{y}_{1}+d \tilde{y}_{1 / 2}\right)-$ $d\left(x_{1 / 2} y\right)=0$ for $d \tilde{y}_{1}+d \tilde{y}_{1 / 2} \in \operatorname{Ker} A_{1 / 2}$ and $d \in \operatorname{Ker} A_{1 / 2}$. From (1) we now easily deduce

(2) $A_{d}(\mathfrak{A}) \mathfrak{A}_{i}=0$ for $i=1, \frac{1}{2}$. Let $x \in \mathfrak{A}_{0}$ and $t \in \mathbf{R}$. We form $W=$ $\exp t A_{d}(x)$ and by (2) get for $z=z_{1}+z_{1 / 2}+z_{0} \in \mathfrak{A}$ immediately $W z=z_{1}$ $+z_{1 / 2}+W_{0} z_{0}, W_{0}:=\left.W\right|_{\mathfrak{x}_{0}} \in \operatorname{Aut}\left(K_{0}, \eta_{0}\right)$.

Further $W_{0}\left(p_{0} \cdot x_{1 / 2}^{2}\right)=p_{0} \cdot x_{1 / 2}^{2}$ for all $x_{1 / 2} \in \mathfrak{A}_{1 / 2}$ because of $A_{d}\left(\mathfrak{A}_{0}\right) \tilde{\mathfrak{A}}_{0}=$ 0. Hence $W \in$ Aut $\mathfrak{A}_{p}$ and $W\left(K_{1}+K_{0}\right)=K_{1}+K_{0}$. By Theorem 1.8, we conclude $W \in \operatorname{Aut}(K, \eta)$ and $A_{d}(x) \in \operatorname{Lie} \operatorname{Aut}(K, \eta)$ for all $x \in \mathfrak{A}$. This shows $d \in \mathfrak{S}$.

COROllary 7.6. (a) $A_{1 / 2}: \mathfrak{A}_{0}(c) \rightarrow$ End $\mathfrak{A}_{1 / 2}(c)$ is injective,

(b) $\mathfrak{A}_{0}(c)$ is generated (as a vector space) by $M=\left\{c_{0} \cdot x_{1 / 2}^{2} ; x_{1 / 2} \in \mathfrak{U}_{1 / 2}(c)\right\}$.

REMARK 7.7. Using the above methods and results for $p=c$ it can be 
shown, [3], that

(a) The closure of $K_{0}$ equals the set of finite sums of elements of $M$.

(b) The closure of $K$ equals the set of finite sums of squares in $\mathfrak{A}_{c}$.

This result has also been obtained in a recent paper of $O$. Rothaus [11].

8. Inductive construction of homogeneous regular cones. In this paragraph we unfold how to construct every homogeneous regular cone in a unique way out of lower dimensional ones.

Here, for a finite-dimensional vector space $V^{\prime}$ over $\mathbf{R}$ and a positive definite bilinear form $\rho$ on $V^{\prime}$, we put $\operatorname{Sym}\left(V^{\prime}, \rho\right):=\left\{R \in\right.$ End $V^{\prime} ; R^{\rho}=$ $R$ \}.

The "data" needed for the construction are numbered (D.x).

Simplifying notations and definitions are denoted by (N.x).

Most of the purely technical calculations have been omitted.

(D.1) Let $F_{1}$ and $F_{0}$ be triples of $\mathcal{F}$.

All that is derived for $F_{i}$ is marked by $F_{i}$ or-if no confusion can arise-just by $i$, e.g. $h_{i}, \sigma_{i}, \ldots$.

We abbreviate $p_{i}:=e_{F_{i}}$. Here we use the letter $p$ to point out that the element $e_{F}$ is not automatically the unit of $\widetilde{S}_{F}$ (which is denoted by $c$ ) where $F$ is the triple which we are going to construct in Theorem 8.2. To assure that $p_{1}$ is the unit $c=c_{1}$ of $\widetilde{S}_{F}$ we shall have to impose condition (D.6).

(D.2) Let $V^{\prime}$ be a finite dimensional vector space over $\mathbf{R}$ and $\rho$ be a positive definite bilinear form on $V^{\prime}$. Further, for $i=0,1$ let mappings $U_{i}: V_{i} \rightarrow$ $\operatorname{Sym}\left(V^{\prime}, \rho\right)$ be given such that $U_{i}\left(p_{i}\right)=\frac{1}{2} \operatorname{Id}_{V^{\prime}}$ and $U_{i}\left(x_{i}\right)$ is positive definite with respect to $\rho$ for all $x_{i} \in K_{i}^{\sigma_{i}}$.

(D.3) For $i=0,1$ let $\Phi_{i} \subset \mathrm{GL} V_{i} \times \mathrm{GL} V^{\prime}$ be Lie-subgroups such that for their projections $\Phi_{i i}\left(\right.$ resp. $\left.\Phi_{i 1 / 2}\right)$ of $\Phi_{i}$ on GL $V_{i}$ (resp. GL $\left.V^{\prime}\right)$ and all $\left(W_{i i}\right.$, $W_{i 1 / 2} \in \Phi_{i}$ we have

(a) $U_{i}\left(W_{i i}^{\sigma_{i}} x_{i}\right)=W_{i 1 / 2}^{\rho} U_{i}\left(x_{i}\right) W_{i 1 / 2}$ for all $x_{i} \in V_{i}$.

(b) $\Phi_{i i} \in \operatorname{Aut}\left(K_{i}, \eta_{i}\right)$ and $\Phi_{i i}$ operates transitively on $K_{i}$.

(c) $\left[W_{11 / 2}, U_{0}\left(x_{0}\right)\right]=0$ for all $x_{0} \in V_{0}$,

(d) $\left[W_{01 / 2}, U_{1}\left(x_{1}\right)\right]=0$ for all $x_{1} \in V_{1}$.

(N.1) Under the assumption of (D.1) and (D.2), we put $V:=V_{1} \oplus V^{\prime} \oplus$ $V_{0}$ and define $\sigma$ to be the sum of $\sigma_{1}, \rho$ and $\sigma_{0}$. Further we define an algebra $\mathfrak{A}$ on $V$ by

$$
\begin{aligned}
\left(w_{1}+w^{\prime}+w_{0}\right) & \left(v_{1}+v^{\prime}+v_{0}\right) \\
:= & {\left[A_{F_{1}}\left(w_{1}\right) v_{1}+a_{1}\left(w^{\prime}, v^{\prime}\right)\right] } \\
& +\left[U_{1}\left(w_{1}\right) v^{\prime}+U_{1}\left(v_{1}\right) w^{\prime}+U_{0}\left(w_{0}\right) v^{\prime}+U_{0}\left(v_{0}\right) w^{\prime}\right] \\
& +\left[A_{F_{0}}\left(w_{0}\right) v_{0}+a_{0}\left(w^{\prime}, v^{\prime}\right)\right]
\end{aligned}
$$


where $a_{i}\left(w^{\prime}, v^{\prime}\right)$ is given by the equation $\sigma_{i}\left(a_{i}\left(w^{\prime}, v^{\prime}\right), x_{i}\right)=\rho\left(U_{i}\left(x_{i}\right) w^{\prime}, v^{\prime}\right)$.

(N.2) In what follows we write $V_{1 / 2}$ instead of $V^{\prime}$. Further instead of $\sigma_{i}$ or $\rho$ we write $\sigma$. The product in $\mathfrak{A}$ is denoted by $x y$ or $x \cdot y$ and we abbreviate as usual $x \cdot y z:=x(y z)$. We easily see $a_{i}\left(x_{1 / 2}, y_{1 / 2}\right)=p_{i} \cdot x_{1 / 2} y_{1 / 2}$. The left multiplications in $\mathfrak{A}$ are denoted by $A(x)$.

LEMMA 8.1. With (D.1), (D.2), (D.3) and (N.1), (N.2) we have

(a) $\mathfrak{A}$ is a commutative algebra with unit $e:=p_{1}+p_{0}$.

(b) $V_{i}=\left\{x \in V ; p_{i} x=x\right\}, i=0,1$.

(c) $\sigma(u v, w)=\sigma(u, v w)$ for all $u, v, w \in \mathfrak{A}$.

(d) $\left[U_{1}\left(x_{1}\right), U_{0}\left(x_{0}\right)\right]=0$ for all $x_{1} \in V_{1}, x_{0} \in V_{0}$.

(e) Let $i, j=0,1, i \neq j$ and $x_{1 / 2}, y_{1 / 2} \in V_{1 / 2}, x_{i} \in V_{i}, W_{i 1 / 2} \in \Phi_{i 1 / 2}$ then

$$
\begin{gathered}
p_{j}\left(U_{i}\left(x_{i}\right) x_{1 / 2} \cdot y_{1 / 2}\right)=p_{j}\left(x_{1 / 2} \cdot U_{i}\left(x_{i}\right) y_{1 / 2}\right), \\
p_{j}\left(W_{i 1 / 2} x_{1 / 2} \cdot y_{1 / 2}\right)=p_{j}\left(x_{1 / 2} \cdot W_{i 1 / 2}^{o} y_{1 / 2}\right) .
\end{gathered}
$$

(f) For all $i, j=0,1, i \neq j, x_{1 / 2} \in V_{1 / 2}, x_{1} \in K_{1}^{\sigma}, x_{0} \in K_{0}^{o}$ we have

$$
p_{j}\left(U_{i}\left(x_{i}\right) x_{1 / 2} \cdot x_{1 / 2}\right)=0 \Leftrightarrow x_{1 / 2}=0 \text {. }
$$

(g) Under the same additional assumptions as in (f) we have

$$
\begin{array}{ll}
p_{0}\left(U_{1}\left(x_{1}\right) x_{1 / 2} \cdot x_{1 / 2}\right) \in \bar{K}_{0} & \left(\text { closure of } K_{0}\right), \\
p_{1}\left(U_{0}\left(x_{0}\right) x_{1 / 2} \cdot x_{1 / 2}\right) \in \bar{K}_{1} & \left(\text { closure of } K_{1}\right) .
\end{array}
$$

Proof. For (d) we note $\left[\frac{1}{2} W_{11 / 2}^{\sigma} W_{11 / 2}, U_{0}\left(x_{0}\right)\right]=0$ because of (D.3). Further, $U_{1}\left(W_{11}^{o} p_{1}\right)=\frac{1}{2} W_{11 / 2}^{\sigma} W_{11 / 2}$ in addition to (D.3)(b) proves (d). For (g) we have $\sigma\left(x_{0}, p_{0}\left(U_{1}\left(x_{1}\right) x_{1 / 2} \cdot x_{1 / 2}\right)\right)=\sigma\left(U_{0}\left(x_{0}\right) U_{1}\left(x_{1}\right) x_{1 / 2}, x_{1 / 2}\right)$. Now $U_{1}\left(x_{1}\right) U_{0}\left(x_{0}\right)$ is positive definite because of (d) and (D.2).

(N.3) For $x=x_{1}+x_{1 / 2}+x_{0} \in V$ and $U_{1}\left(x_{1}\right) \in$ GL $V_{1 / 2}$ we put

(a) $k_{1 / 2}(x):=\left[U_{1}\left(x_{1}\right)\right]^{-1} x_{1 / 2}$ and $k_{0}(x):=x_{0}-\frac{1}{2} p_{0}\left(x_{1 / 2} \cdot k_{1 / 2}(x)\right)$,

(b) $K:=\left\{x \in V ; x_{1} \in K_{1}^{\sigma}, k_{0}(x) \in K_{0}\right\}$,

(c) $\eta(x):=\tilde{\eta}_{1}\left(x_{1}\right) \eta_{0}\left(k_{0}(x)\right), x \in K$, where $\tilde{\eta}_{1}$ is defined from $F_{1}$ by [5a, (1.19)],

(d) $\Phi_{0}^{\prime}:=\left\{\left(\mathrm{Id}, W_{01 / 2}, W_{00}\right) \in \mathrm{GL} V ;\left(W_{01 / 2}, W_{00}\right) \in \Phi_{0}\right\}$, $\Phi_{1}^{\prime}:=\left\{\left(W_{11}, W_{11 / 2}\right.\right.$, Id $\left.) \in \mathrm{GL} V ;\left(W_{11}, W_{11 / 2}\right) \in \Phi_{1}\right\}$.

The following theorem uses [10, IV, Theorem 27].

TheOREM 8.2. With (D.1), (D.2), (D.3) and (N.1), (N.2), (N.3) we have

(a) $K$ is a homogeneous regular cone,

(b) $F:=\langle K, \eta, e\rangle$ is a triple of $\mathcal{F}$.

More precisely we have $\left(p:=p_{1}\right)$

(c) The group generated by $\Phi_{1}^{\prime \sigma} \cup\left\{\exp A_{p}\left(x_{1 / 2}\right) ; x_{1 / 2} \in V_{1 / 2}\right\} \cup \Phi_{0}^{\prime}$ is contained in $\operatorname{Aut}(K, \eta)$ and operates transitively on $K$.

(d) $K=\cup_{x_{1 / 2} \in V_{1 / 2}} \exp A_{p}\left(x_{1 / 2}\right)\left(K_{1}^{\sigma}+K_{0}\right)\left(\right.$ for $K_{i}$ see (D.1)).

(e) $\sigma_{F}=\sigma, \mathfrak{U}=\mathfrak{U}_{F}$. 
Proof. First note that $K$ is a regular convex cone by [10, IV, Theorem 27]. Further (d) follows easily from the definition of $K$. Hence only (b), (c), and (e) are to be proved. As to (c) it is easy to check $\left[A_{p}\left(x_{1 / 2}\right), A_{p}\left(y_{1 / 2}\right)\right]=0$; hence $W:=\exp A_{p}\left(x_{1 / 2}\right)$ maps $K$ onto $K$. Further $k_{0}(W x)=k_{0}(x)$, so $A_{p}\left(x_{1 / 2}\right) \in \operatorname{Lie} \operatorname{Aut}(K, \eta)$. Now it is not hard to verify $W^{\sigma} A_{p}\left(x_{1 / 2}\right) W^{\sigma-1}=$ $A_{p}\left(W^{-1} x_{1 / 2}\right)$ for $W \in \Phi_{1}^{\prime}$ and $W A_{p}\left(x_{1 / 2}\right) W^{-1}=A_{p}\left(W x_{1 / 2}\right)$ for $W \in \Phi_{0}^{\prime}$. From this (c) follows. We show (b) and have only to check (1.3) and (1.4). For (1.4), we denote by $\partial X$ the boundary of a set $X \subset V$ and let $x^{(n)} \in K$, such that $x^{(n)} \rightarrow x \in \partial K$. Then we have $x_{i}^{(n)} \rightarrow x_{i}$. We first demonstrate that $\eta_{0}\left(k_{0}\left(x^{(n)}\right)\right)$ is bounded away from zero by a constant $R>0$. For this we note that the sequence $z_{n}:=\frac{1}{2} p_{0}\left(\left[U_{1}\left(x_{1}^{(n)}\right)\right]^{-1} x_{1 / 2}^{(n)} \cdot x_{1 / 2}^{(n)}\right)$ is bounded; for if this were false we could choose a norm $|\cdot|$ on $V$ and assume that $\left|z_{n}\right|>n$ and that $\left|z_{n}\right|^{-1} z_{n}$ converges. Then $z:=\lim \left|z_{n}\right|^{-1} z_{n} \in \bar{K}_{0}$ and $\lim \left|z_{n}\right|^{-1}\left(x_{0}^{(n)}-z_{n}\right) \in \bar{K}_{0}$. But $\lim \left|z_{n}\right|^{-1} x_{0}^{(n)}=0$, hence $\pm z \in \bar{K}_{0}$ and $z=0$ by the regularity of $K_{0}$. This contradicts $|z|=1$ proving that $z_{n}$ is bounded. But then $k_{0}\left(x^{(n)}\right)$ is bounded and there exists an $\alpha>0$ such that $\alpha p_{0}-k_{0}\left(x^{(n)}\right) \in K_{0}$ for all $n$. From this we conclude $0<\eta_{0}\left(\alpha p_{0}\right)=\eta_{0}\left(k_{0}\left(x^{(n)}\right)+\alpha p_{0}-k_{0}\left(x^{(n)}\right)\right)<\eta_{0}\left(k_{0}\left(x^{(n)}\right)\right)$ by [5, Lemma 3.4]. We are now ready to demonstrate (1.4). If $x_{1}^{(n)} \rightarrow x_{1} \in K_{1}^{o}$, then $k_{0}\left(x^{(n)}\right) \rightarrow k_{0}(x) \in \partial K_{0}$ for otherwise $x \in K$ by definition of $K$. Hence $\eta\left(x^{(n)}\right)$ $\rightarrow+\infty$. Now assume $x_{1} \in \partial K_{1}^{\sigma}$, then $\eta_{1}\left(x_{1}^{(n)}\right) \rightarrow+\infty$. Because of $\eta_{0}\left(k_{0}\left(x^{(n)}\right)\right)$ $>R>0$ the assertion follows. To verify (1.3) it is easy to show $\sigma(u, v)=$ $\left.\Delta_{x}^{u} \Delta_{x}^{v} \log \eta(x)\right|_{x=e}$. Hence from (1.13) we deduce (1.3) by (c). Further we get $\sigma=\sigma_{F}$. Finally $\mathfrak{A}=\mathfrak{A}_{F}$ is seen by a straightforward computation.

REMARK 8.3. Up to (N.3) all definitions and results are symmetric in $K_{1}$ and $K_{0}$. If in (N.3) we had choosen the "dual" definition in interchanging 1 and 0 then the construction would have produced $K^{\sigma}$. The case $V_{1}=\mathbf{R}$, $K_{1}=\mathbf{R}^{+}$has been investigated along these lines in [9].

Now we impose further conditions to specialize to the situation looked at in the foregoing paragraphs.

(D.4) Assume $K_{1}=K_{1}^{\sigma}$.

Then by Theorem 4.7 the algebra $\mathfrak{A}_{F_{1}}$ on $V_{1}$ is a Jordan-algebra with unit $p=p_{1}$.

(D.5) Assume that $2 U_{1}: \mathfrak{A}_{F_{1}} \rightarrow \operatorname{Sym}\left(V^{\prime}, \rho\right)$ is a homomorphism of Jordan-algebras. Assume further that $U_{0}$ is injective.

REMARK 8.4. (a) It is not hard to see that under the additional assumptions (D.4) and (D.5) we may replace $\Phi_{1}^{\prime}$ by the group $\Phi_{1}^{\prime \prime}$ that is generated by $\left\{\exp A\left(x_{1}\right) ; x_{1} \in V_{1}\right\}$.

(b) Further, from Theorem 8.2 we get $V_{1}=\mathfrak{A}_{1}\left(p_{1}\right) \subset \Im_{F}$.

(D.6) Assume $\delta^{p}=\operatorname{Lie} \operatorname{Aut}(K, \eta)$.

REMARK 8.5. (a) Instead of (D.6), which involves $K$ and $\eta$ in a rather complicated way, we could have imposed the following condition which comes out of Theorem 3.4. 
(D.6) The set of $x_{1 / 2} \in \mathfrak{A}_{1 / 2}$ which satisfy (b)(i) and (b)(ii) of Theroem 3.4 consists only of 0 .

(b) From Theorem 7.5 we deduce $V_{1}=\mathfrak{S}_{F}$.

We are now ready to prove the Construction Theorem.

For the convenience of the reader we repeat all assumptions in full detail. For simplicity we are again using the letters $F_{1}$ and $F_{0}$. We hope that this will help the reader refer more easily to the foregoing sections.

TheORem 8.6 (CONSTRUCTION TheOREM). I. We assume the following conditions and notations:

(C.1) $F_{1}$ and $F_{0}$ are triples of $\mathcal{F}$.

(C.2) $V_{1 / 2}$ is a finite-dimensional vectorspace over $\mathbf{R}$ and $\sigma_{1 / 2}$ a positive-definite bilinear form of $V_{1 / 2}$.

(C.3) $K_{F_{1}}=K_{F_{1}}^{\sigma_{F_{1}}}$.

(C.4) $2 U_{1}: V_{F_{1}} \rightarrow \operatorname{Sym}\left(V_{1 / 2}, \sigma_{1 / 2}\right)$ is a unital homomorphism of the formallyreal Jordan-algebra $\mathfrak{A}_{F_{1}}$ into $\operatorname{Sym}\left(V_{1 / 2}, \sigma_{1 / 2}\right)$.

(C.5) $U_{0}: V_{F_{0}} \rightarrow \operatorname{Sym}\left(V_{1 / 2}, \sigma_{1 / 2}\right)$ is an injective linear mapping such that $U_{0}\left(x_{0}\right)$ is positive-definite with respect to $\sigma_{1 / 2}$ for all $x_{0} \in K_{0}^{\sigma_{F_{0}}}$. Further, we assume $U_{0}\left(e_{F_{0}}\right)=\frac{1}{2} \mathrm{Id}$.

(C.6) There exists a Lie-subgroup $\Phi_{0}$ of GL $V_{F_{0}} \times \mathrm{GL} V_{1 / 2}$ such that for its projections $\Phi_{00}\left(\right.$ resp. $\left.\Phi_{01 / 2}\right)$ on GL $V_{F_{0}}\left(\right.$ resp. GL $\left.V_{1 / 2}\right)$ we have

(a) $\Phi_{00} \subset \operatorname{Aut}\left(K_{F_{0}}, \eta_{F_{0}}\right)$ and $\Phi_{00}$ operates transitively on $K_{F_{0}}$.

(b) For all $\left(W_{00}, W_{01 / 2}\right) \in \Phi_{0}$ and all $x_{i} \in V_{F_{i}}, i=0,1$ we have

$U_{0}\left(W_{00}^{\sigma}{ }^{o} x_{0}\right)=W_{0}^{o} \gamma_{1 / 2} U_{0}\left(x_{0}\right) W_{01 / 2}$,

$\left[U_{1}\left(x_{1}\right), W_{01 / 2}\right]=0$.

(C.7) The set of all $x_{1 / 2} \in V_{1 / 2}$ satisfying the following two conditions consists only of 0 .

(a) $U_{0}\left(h_{F_{0}}\left(y_{0}\right)\right) U_{0}\left(y_{0}\right) x_{1 / 2}=\frac{1}{4} x_{1 / 2}$ for all $y_{0} \in K_{F_{0}}$,

(b) $a_{0}\left(y_{1 / 2} \cdot U_{1}\left(a_{1}\left(x_{1 / 2} \cdot U_{1}\left(y_{1}\right) y_{1 / 2}\right)\right) y_{1 / 2}\right)=a_{0}\left(y_{1 / 2} \cdot U_{0}\left(a_{0}\left(y_{1 / 2} \cdot\right.\right.\right.$ $\left.\left.U_{1}\left(y_{1}\right) y_{1 / 2}\right)\right) x_{1 / 2}$ for all $y_{1} \in V_{F_{1}}$ and all $y_{1 / 2} \in V_{1 / 2}$.

(Here $a_{i}$ is defined by $\sigma_{F_{i}}\left(x_{i}, a_{i}\left(x_{1 / 2}, y_{1 / 2}\right)\right)=\sigma_{1 / 2}\left(U_{i}\left(x_{i}\right) x_{1 / 2}, y_{1 / 2}\right)$.)

II. Conclusions. Denote by $\mathcal{H} \mathcal{K}$ the class of tuples $X=\left(F_{1}, F_{0}, V_{1 / 2}, \sigma_{1 / 2}\right.$, $\left.U_{1}, U_{0}\right)$ which satisfy (C.1) to (C.7) and define $\chi(X)$ to be the triple of $\mathscr{F}$ which is constructed out of $X$ in Theorem 8.2. Then we have

(a) $\chi$ is a bijection of $\mathcal{H} \mathcal{K}$ onto $\mathcal{F}$.

(b) For $X=\left(F_{1}, F_{0}, V_{1 / 2}, \sigma_{1 / 2}, U_{1}, U_{0}\right)$ we get

$$
\chi(X)_{i}=F_{i}, \quad i=0,1,
$$

if in (2.3) we choose $p$ to be the unit $c$ of $\mathfrak{S}_{X(X)}$. Further $\left(\mathfrak{A}_{X(X)}\right)_{i}=V_{i}, i=0, \frac{1}{2}$, 1 and $U_{i}\left(x_{i}\right)=\left.A_{\chi(X)}\left(x_{i}\right)\right|_{V_{1 / 2}}, i=0,1$. 
(c) The inverse mapping of $\chi$ is given by assigning to each triple $F$ of $\mathcal{F}$ the tuple $X=\left(F_{1}, F_{0}, V_{1 / 2}, \sigma_{1 / 2}, U_{1}, U_{0}\right)$, where

(1) $V_{1 / 2}$ denotes the Peirce- $\frac{1}{2}$-space of $\mathfrak{A}_{F}$ with respect to $c$,

(2) $\sigma_{1 / 2}$ is the restriction of $\sigma_{F}$ to $V_{1 / 2}$,

(3) $F_{i}$ is given by (2.3), defined with respect to the unit $c$ of $\mathfrak{S}_{F}$.

(4) $U_{i}\left(x_{i}\right):=\left.A_{F}\left(x_{i}\right)\right|_{V_{i}}, x_{i} \in V_{i}, i=0,1$.

Proof. First note that $\chi$ is well defined by Theorem 8.2 . We denote by $\kappa$ the mapping defined in (c) and are going to prove that $\kappa(X)$ is in $\mathcal{H} \mathcal{K}$. We have to check (C.1) to (C.7). But (C.1) follows from Corollary 3.8 and (C.2) is obvious. Further, (C.3) is clear because of Theorem 1.6. From [2, Satz 2.1], and Theorem 1.2(b) we get (C.4) for $U_{1}$. Next we state (C.5) for $U_{0}$, as Corollary 7.6 shows. To verify (C.6) we define $\Phi_{0}:=\left.\Delta_{0}^{c}\right|_{M}, M:=\mathfrak{A}_{1 / 2}(c)+$ $\mathfrak{A}_{0}(c)$, and refer to Lemma 3.6 and Theorem 3.7. Finally, (C.7) is just the statement of Theorem 3.4. To prove the theorem, it is enough to show that $\kappa$ is the inverse mapping for $\chi$.

Let us start first with a triple $F$ of $\mathscr{F}, F=\langle K, \eta, e\rangle$. We split $\mathfrak{A}$ with respect to the unit $c$ of $\subseteq=\Im_{F}$. We compare Lemma 2.1 with part (c) of (N.3) and see that the function, constructed out of $\kappa(F)$ by (N.3) coincides with the original $\eta$. Similarly Theorem 1.8 and Theorem 8.2(d) show that the cone, constructed for $\chi(F)$ equals $K$. Obviously $e=c_{1}+c_{0}$ and $\chi(\kappa(F))$ is proved for all triples $F$ of $\mathscr{F}$. Let us start now with a tuple $X$ of $\mathcal{H} \mathscr{K}$. Then by Remark 8.5(b) we have $V_{1}=\mathfrak{S}_{\chi(X)}$, hence $p_{1}=e_{F_{1}}$ is the unit $c$ of $\mathfrak{S}_{X(X)}$. By definition of $\kappa$ we have to split $\mathfrak{A}=\mathfrak{A}_{\chi(X)}$ with respect to $c=p_{1}$. But, using (2.3) and (N.3), we obviously get $\kappa(\chi(X))=X$ which finishes the proof.

\section{REFERENCES}

1. H. Braun and M. Koecher, Jordan-Algebren, Springer-Verlag, Berlin, 1966.

2. J. Dorfmeister, Zur Konstruktion homogener Kegel, Math. Ann. 216 (1975), 79-96.

3. __ Eine Theorie der homogenen, regulären Kegel, Dissertation, Univ. Münster, 1974.

4. J. Dorfmeister and M. Koecher, Relative Invarianten und nichtassoziative Algebren, Math. Ann. 228 (1977), 147-186.

5. __, Reguläre Kegel, Jber. Deutsch. Math.-Verein (to appear).

5a. J. Dorfmeister, Peirce-Zerlegungen und Jordan-Strukturen zu homogenen Kegeln (preprint).

6. N. Jacobson, Structure and representations of Jordan algebras, Amer. Math. Soc. Colloq. Publ., vol. 39, Amer. Math. Soc., Providence, R. I., 1968.

7. M. Koecher, Jordan-algebras and their applications, Lecture Notes, Univ. of Minnesota, Minneapolis, 1962.

8. __ Eine Konstruktion von Jordan-Algebren, Manuscripta Math. 23 (1978), 387-425.

9. O. Rothaus, The construction of homogeneous convex cones, Ann. of Math. (2) 83 (1966), $358-376$.

10. __ Automorphisms of Siegel domains, Trans. Amer. Math. Soc. 162 (1971), 351-382.

11. __ Ordered Jordan algebras (preprint). 
12. E. Vinberg, The Morozov-Borel theorem for real Lie groups, Soviet Math. Dokl. 2 (1961), $1416-1419$.

13. The theory of convex homogeneous cones, Trans. Moscow Math. Soc. 12 (1963), $340-403$.

14. The structure of the group of automorphisms of a homogeneous convex cone, Trans. Moscow Math. Soc. 13 (1965), 63-93.

School of Mathematics, Institute for Advanced Study, Princeton, New Jersey 08540

Fachbereich Mathematik, Westfälische Wilhelms UNiVersität MüNSTer, D-44 MüNSTER, Federal RePublic of Germany (Current address) 